MANUAL OF

MATERIALS DATA RELEASE MEMORANDA

\title{
SUPPLEMENT I
}

NERVA Program

\section{NOTICE}

This report was prepared as an account of work sponsored by the United States Government. Neither the United States nor the United States Energy Research and Development Administration, nor any of their employees, nor any of their contractors, subcontractors, or their employees, makes any warranty, express or implied, or assumes any legal lability or responsibility for the accuracy, completeness or usefulness of any information, apparatus, product or process disclosed, or represents that its use would not infrnge privately owned rights.

$$
\text { Pacto }
$$

K. Sato

Manager, Engineering Staff

Engineering Operations

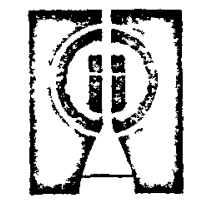

\section{Contract SNP-1}

JULY 1971 


\section{DISCLAIMER}

This report was prepared as an account of work sponsored by an agency of the United States Government. Neither the United States Government nor any agency Thereof, nor any of their employees, makes any warranty, express or implied, or assumes any legal liability or responsibility for the accuracy, completeness, or usefulness of any information, apparatus, product, or process disclosed, or represents that its use would not infringe privately owned rights. Reference herein to any specific commercial product, process, or service by trade name, trademark, manufacturer, or otherwise does not necessarily constitute or imply its endorsement, recommendation, or favoring by the United States Government or any agency thereof. The views and opinions of authors expressed herein do not necessarily state or reflect those of the United States Government or any agency thereof. 


\section{DISCLAIMER}

Portions of this document may be illegible in electronic image products. Images are produced from the best available original document. 
MANUAL OF MATERIALS DATA RELEASE MEMORANDA, SUPPLEMENT I, 15 JULY 1971 LISTING OF INCLUDED DRM'S

\begin{tabular}{|c|c|c|c|c|c|c|c|c|}
\hline MATERIAL & CONDITION & FORM & DIRECTION & PROPERTIES & $\begin{array}{l}\text { TEST } \\
\text { IEMPERATURES, }{ }^{\circ} \mathrm{F}\end{array}$ & $\begin{array}{l}\text { DATA } \\
\text { CATEGORY }\end{array}$ & $\begin{array}{l}\text { IS SUE } \\
\text { DATE }\end{array}$ & $\begin{array}{l}\text { DRM } \\
\text { NO. } \\
\end{array}$ \\
\hline Ti-5A1-2.5Sn ELI & ANNEALED & BAR & $A, R B$ & FATIGUE & $-423,-320, R T$ & $c$ & $4-21-71$ & 04.05 \\
\hline ALLOY A-286 & ANNEALED \& AGED & SHEET & $F$ & FATIGUE & $-423,-320, R T$ & $c$ & $4-21-71$ & 05.04 \\
\hline Alloy A-286 & SOL. TREATED \& AGED & FORGINGS & TANG & MECHANICAL & $-423,-110, \mathrm{RT}$ & B & $5-24-71$ & 05.05 \\
\hline AG CARB-101 & $\begin{array}{l}\text { IRRADIATED \& } \\
\text { ANNEALED }\end{array}$ & $N / A$ & N/A, & MECHANICAL & RT & $\begin{array}{l}\text { C } \\
\star\end{array}$ & $7-2-71$ & 06.04 \\
\hline
\end{tabular}

FOR ABBREVIATIONS, SEE PAGE 3 OF DRM MANUAL

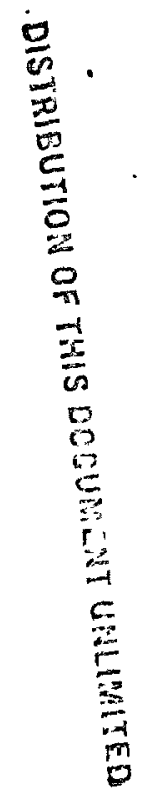




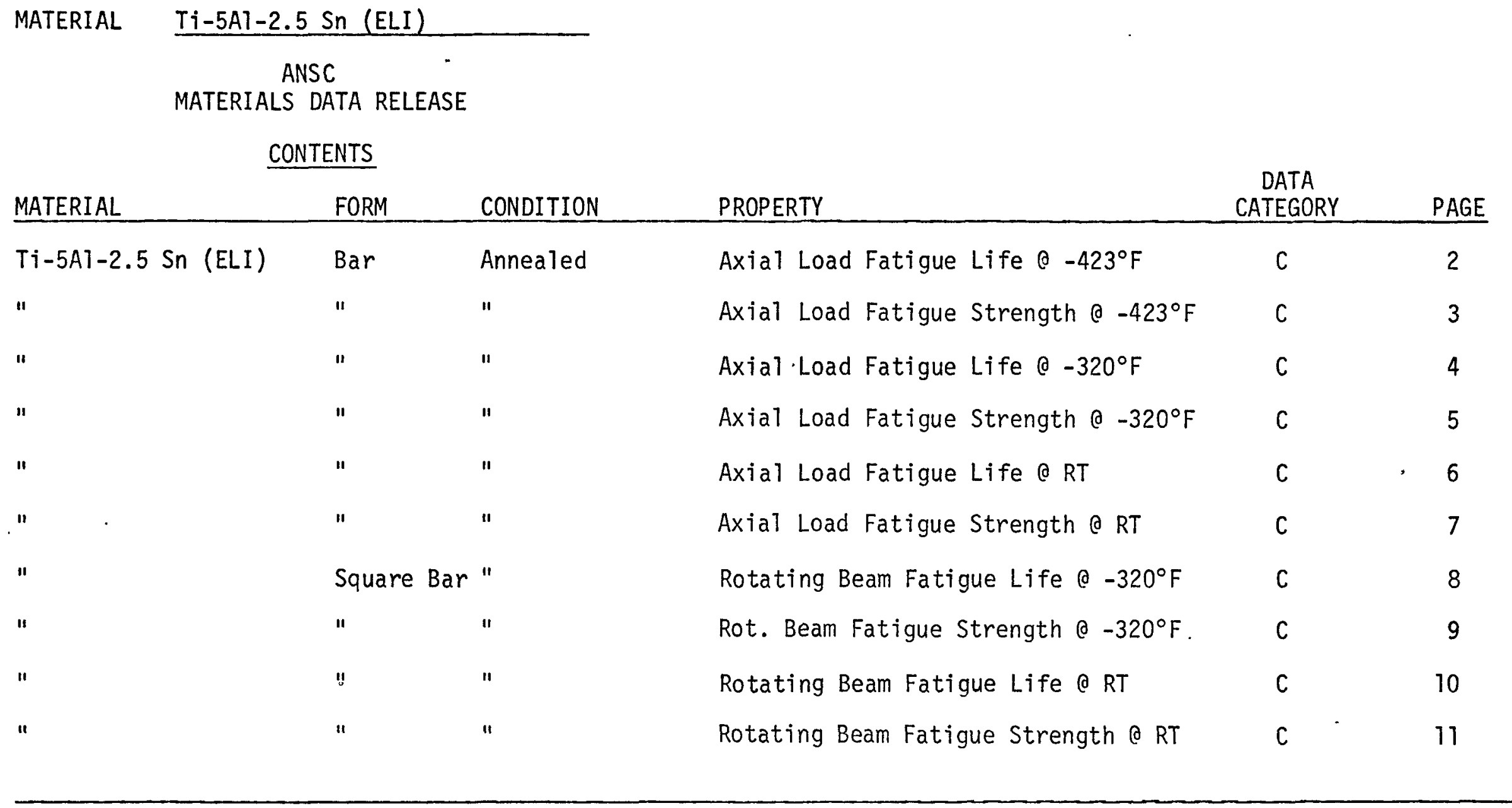

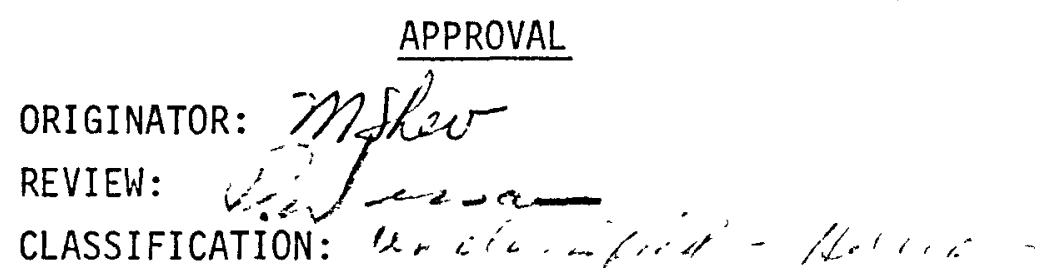


MATERIAL

$\underline{T i-5 A]-2.5 \mathrm{Sn} \text { (ELI) }}$

SPECIFICATIONS

RB $0170-79$

\section{AXIAL LOAD FATIGUE LIFE 0}

\section{PROPERTY}

FORM

DIRECTION
BAR

CONDITION

ANNEALED

\section{AXIAL LOAD FATIGUE LIFE $0-423$}

\begin{tabular}{|c|c|c|c|c|c|c|c|c|c|c|}
\hline \multirow[b]{2}{*}{$\begin{array}{c}\text { STRESS } \\
\text { KSI }\end{array}$} & \multicolumn{6}{|c|}{ LOG OF CYCLES } & \multicolumn{2}{|c|}{ NO. OF CYCLES $\times 10^{3}$} & \multirow[b]{2}{*}{$\begin{array}{l}\text { DATA } \\
\text { CATEGORY }\end{array}$} & \multirow[b]{2}{*}{$\begin{array}{c}\text { SOURCE } \\
\text { REFERENCE }\end{array}$} \\
\hline & MEAN & $\begin{array}{l}\text { STANDARD } \\
\text { ERROR } \\
\text { OF } \\
\text { ESTIMATE } \\
\text { se } \\
\end{array}$ & $\begin{array}{c} \\
\text { EFFECTIVE } \\
\text { SAMPLE } \\
\text { SIZE } \\
\mathrm{m}\end{array}$ & $\begin{array}{c}\text { DEGREES } \\
\text { OF } \\
\text { FREEDOM } \\
\text { FOR } \\
\text { se } \\
\end{array}$ & $k$ & $\begin{array}{l}\text { LOWER } \\
\text { 99/95 } \\
\text { LIMIT }\end{array}$ & MEAN & $\begin{array}{c}\text { DESIGN } \\
\text { ALLOWABLE }\end{array}$ & & \\
\hline 200 & 4.26 & 0.231 & 10.3 & 26 & 3.24 & 3.51 & 18 & 3 & C & 1 \\
\hline 190 & 4.35 & 0.231 & 10.3 & 26 & 3.24 & 3.61 & 23 & 4 & c & 1 \\
\hline 180 & 4.48 & 0.231 & 10.4 & 26 & 3.24 & 3.74 & 30 & 5 & c & 1 \\
\hline 170 & 4.67 & 0.231 & 10.5 & 26 & 3.24 & 3.93 & 47 & 8 & c & 1 \\
\hline 160 & 4.92 & 0.231 & 10.5 & 26 & 3.24 & 4.17 & 82 & 15 & c & 1 \\
\hline 150 & 5.23 & 0.231 & 10.5 & 26 & 3.24 & 4.48 & 171 & 30 & c & 1 \\
\hline 140 & 5.64 & 0.231 & 10.3 & 26 & 3.24 & 4.89 & .438 & 78 & c & 1 \\
\hline 130 & 6.17 & 0.231 & 10.3 & 26 & 3.24 & 5.42 & 1486 & 265 & c & 1 \\
\hline
\end{tabular}


DESIGN DATA

\begin{tabular}{|c|c|c|c|c|c|}
\hline MATERIAL & $\mathrm{Ti}-5 \mathrm{Al}-2.5 \mathrm{Sn}$ (ELI) & FORM & BAR & CONDITION & ANNEALED \\
\hline SPECIFICATIONS & RB0170-079 & & DIRECTION & LONGITUDINAL & \\
\hline
\end{tabular}

PROPERTY AXIAL FATIGUE STRENGTH @ $-423^{\circ} \mathrm{F}$

\begin{tabular}{|c|c|c|c|c|c|}
\hline NO. OF CYCLES & $\begin{array}{c}\bar{X} \\
(K S I) \\
\end{array}$ & s & $\begin{array}{c}\text { DESIGN } \\
\text { ALLOWABLE } \\
(K S I)\end{array}$ & $\begin{array}{c}\text { DATA } \\
\text { CATEGORY }\end{array}$ & $\begin{array}{c}\text { SOURCE } \\
\text { REFERENCE }\end{array}$ \\
\hline $10^{6}$ & 133 & 4.9 & 120 & C & 1 \\
\hline$\star 3.16 \times 10^{5}$ & 143.5 & 6.0 & 127.5 & C & 1 \\
\hline $10^{5}$ & 156.5 & 6.8 & 138 & C & 1 \\
\hline $3.16 \times 10^{4}$ & 179 & 13.2 & 149.5 & C & 1 \\
\hline
\end{tabular}

$3.16 \times 10^{5}=10^{5.5}=\operatorname{antilog} 5.5$ 
DRM: $\quad 05$

DESIGN DATA

MATERIAL

$\underline{T i-5 A 7-2.5 \mathrm{Sn}(E L I)}$

FORM BAR

CONDITION

ANNEALED

SPECIFICATIONS

RB $0170-079$

DIRECTION

AXIAL

PROPERTY

AXIAL LOAD FATIGUE LIFE $0-320^{\circ} \mathrm{F}$

\begin{tabular}{|c|c|c|c|c|c|c|c|c|c|c|}
\hline \multirow[b]{2}{*}{$\begin{array}{l}\text { STRESS } \\
\text { (KSI) }\end{array}$} & \multicolumn{6}{|c|}{ LOG OF CYCLES } & \multicolumn{2}{|c|}{ NO. OF CYCLES $\times 10^{3}$} & \multirow[b]{2}{*}{$\begin{array}{l}\text { DATA } \\
\text { CATEGORY }\end{array}$} & \multirow[b]{2}{*}{$\begin{array}{c}\text { SOURCE } \\
\text { REFERENCE }\end{array}$} \\
\hline & MEAN & $\begin{array}{l}\text { STANDARD } \\
\text { ERROR } \\
\text { OF } \\
\text { ESTIMATE } \\
\text { Se } \\
\end{array}$ & $\begin{array}{c}\text { EFFECTIVE } \\
\text { SAMPLE } \\
\text { SIZE } \\
\mathrm{m}\end{array}$ & $\begin{array}{c}\text { DEGREES } \\
\text { OF } \\
\text { FREEDOM } \\
\text { FOR } \\
\text { se } \\
\end{array}$ & $k$ & $\begin{array}{l}\text { LOWER } \\
99 / 95 \\
\text { LIMIT }\end{array}$ & MEAN & $\begin{array}{c}\text { DESIGN } \\
\text { ALLOWABLE }\end{array}$ & & \\
\hline 190 & 4.94 & .231 & 17.4 & 26 & 3.16 & 4.21 & 87 & 16 & $c$ & 1 \\
\hline 180 & 5.04 & .231 & 17.7 & 26 & 3.16 & 4.31 & 111 & 21 & $c$ & 1 \\
\hline 170 & 5.20 & .231 & 18.2 & 26 & 3.16 & 4.47 & 158 & 29 & c & 1 \\
\hline 160 & 5.41 & .237 & 18.5 & 26 & 3.15 & 4.68 & 258 & 48 & c & 1 \\
\hline 150 & 5.70 & .231 & 19.0 & 26 & 3.15 & 4.97 & 503 & 94 & $c$ & 1 \\
\hline 140 & 6.09 & .231 & 19.2 & 26 & 3.15 & 5.34 & 759 & 142 & $c$ & 1 \\
\hline 130 & 6.59 & .231 & 19.4 & 26 & 3.15 & 5.87 & 3926 & 735 & c & 1 \\
\hline 120 & 7.24 & .231 & 19.6 & 26 & 3.15 & 6.54 & 17380 & 3467 & c & 1 \\
\hline
\end{tabular}




\section{DESIGN DATA}

MATERIAL

$T i-5 A 1-2.5 \mathrm{Sn}$ (ELI)

SPECIFICATIONS

PROPERTY

AXIAL FATIGUE STRENGTH $0-320^{\circ} \mathrm{F}$
FORM

BAR

CONDITION

ANNEALED

DIRECTION AXIAL

\begin{tabular}{|c|c|c|c|c|c|}
\hline NO. OF CYCLES & $\begin{array}{c}\bar{X} \\
(K S I) \\
\end{array}$ & $\mathbf{s}$ & $\begin{array}{c}\text { DESIGN } \\
\text { ALLOWABLE } \\
(K S I)\end{array}$ & $\begin{array}{l}\text { DATA } \\
\text { CATEGORY }\end{array}$ & $\begin{array}{c}\text { SOURCE } \\
\text { REFERENCE }\end{array}$ \\
\hline $10^{7}$ & 124 & 3.83 & 114.5 & C & 1 \\
\hline $3.16 \times 10^{6}$ & 132 & 4.28 & 120.5 & C & 1 \\
\hline $10^{6}$ & 142 & 5.90 & 128 & C & 1 \\
\hline $3.16 \times 10^{5}$ & 156 & 8.70 & 136.5 & C & 1 \\
\hline $10^{5}$ & 184 & 15.10 & 149 & C & 1 \\
\hline
\end{tabular}


DESIGN DATA

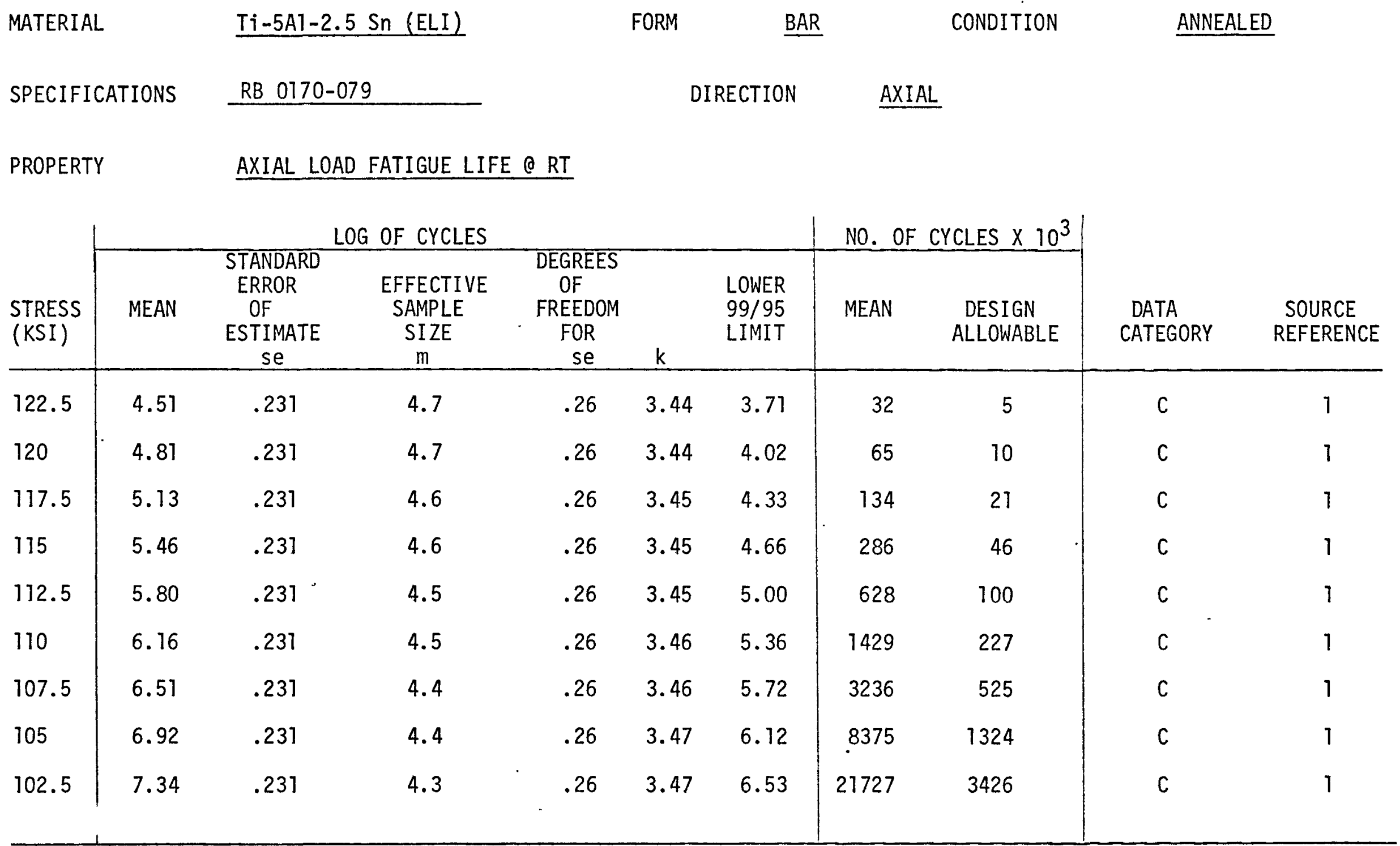


DESIGN DATA

\begin{tabular}{|c|c|c|c|c|c|}
\hline MATERIAL & $T i-5 A T-2.5 \mathrm{Sn}$ (ELI) & FORM & BAR & CONDITION & ANNEALED \\
\hline SPECIFICATIONS & RB0170-079 & & DIRECTION & AXIAL & \\
\hline
\end{tabular}

PROPERTY AXIAL FATIGUE STRENGTH O RT

\begin{tabular}{|c|c|c|c|c|c|}
\hline NO. OF CYCLES & $\begin{array}{c}\bar{X} \\
(K S I) \\
\end{array}$ & $s$ & $\begin{array}{c}\text { DESIGN } \\
\text { ALLOWABLE } \\
(\mathrm{KSI})\end{array}$ & $\begin{array}{l}\text { DATA } \\
\text { CATEGORY }\end{array}$ & $\begin{array}{c}\text { SOURCE } \\
\text { REFERENCE }\end{array}$ \\
\hline $10^{7}$ & 104.4 & 1.45 & 100.3 & C & 1 \\
\hline $3.16 \times 10^{6}$ & 107.6 & 1.60 & 102.7 & C & 1 \\
\hline $10^{6}$ & 111.0 & 1.65 & 105.7 & C & 1 \\
\hline $3.16 \times 10^{5}$ & 114.7 & 1.72 & 109.0 & C & 1 \\
\hline $10^{5}$ & 118.4 & 1.75 & 112.5 & C & 1 \\
\hline $3.16 \times 10^{4}$ & 122.5 & 1.84 & 116.2 & $c$ & 1 \\
\hline
\end{tabular}


DRM: $\quad 05$

DATE: _ 1-71

Page 8 of 14

DESIGN DATA

\begin{tabular}{|c|c|c|c|c|c|c|c|c|c|c|}
\hline MATERIA & & $T i-5 A 1-$ & $\operatorname{Sn}(E L I)$ & & FORM & & SQUARE & & CONDITION & ANNEALED \\
\hline SPECIFI & IIONS & RB0170- & & & DIREC & $10 \mathrm{~N}$ & LONGITU & & & \\
\hline PROPERT & & ROTATIN & EAM FATIGUE & IFE @ & $\mathrm{j}^{\circ} \mathrm{F}$ & & & & & \\
\hline & & & LOG OF CYC & & & & No. 0 & YCLES $\times 10^{3}$ & & \\
\hline $\begin{array}{l}\text { STRESS } \\
\text { (KSI) }\end{array}$ & MEAN & $\begin{array}{l}\text { STANDARD } \\
\text { ERROR } \\
\text { OF } \\
\text { ESTIMATE } \\
\text { se } \\
\end{array}$ & $\begin{array}{l}\text { EFFECTIVE } \\
\text { SAMPLE } \\
\text { SIZE }\end{array}$ & $\begin{array}{c}\text { DEGREES } \\
\text { OF } \\
\text { FREEDOM } \\
\text { FOR } \\
\text { se }\end{array}$ & $k$ & $\begin{array}{l}\text { LOWER } \\
99 / 95 \\
\text { LIMIT }\end{array}$ & MEAN & $\begin{array}{c}\text { DESIGN } \\
\text { ALLOWABLE }\end{array}$ & $\begin{array}{l}\text { DATA } \\
\text { CATEGORY }\end{array}$ & $\begin{array}{l}\text { SOURCE } \\
\text { REFERENCE }\end{array}$ \\
\hline 150 & 4.73 & 0.290 & 8.5 & 21 & 3.37 & 3.75 & 54 & 6 & $C$ & 1 \\
\hline 145 & 5.08 & 0.330 & 9.2 & 21 & 3.36 & 3.95 & 120 & 9 & C & 1 \\
\hline 140 & 5.44 & 0.376 & 9.7 & 21 & 3.35 & 4.19 & 278 & 15 & C & 1 \\
\hline 135 & 5.89 & 0.432 & 10.2 & 21 & 3.33 & 4.45 & 773 & 28 & C & 1 \\
\hline 130 & 6.41 & 0.502 & 10.8 & 21 & 3.32 & 4.74 & 2574 & 55 & C & 1 \\
\hline
\end{tabular}


DESIGN DATA

MATERIAL

Ti-5A1-2.5 Sn (ELI)

FORM SQUARE BAR

CONDITION

ANNEALED

SPECIFICATIONS:

RB $\quad 0170-079$

DIRECTION LONGITUDINAL

PROPERTY

ROTATING BEAM FATIGUE STRENGTH @ -320

NO. OF CYCLES

MEAN
$\bar{X}$
$(K S I)$

DESIGN

ALLOWABLE

DATA

CATEGORY

SOURCE

(KSI)

$10^{5}$

146.0

5.0

126.8

C

1

$3.16 \times 10^{4}$

154.0

6.1

134.0

C 
DRM: $\quad .05$

DATE: $+21-71$

DESIGN DATA

$\begin{array}{llll}\text { MATERIAL } & \text { Ti-5Al-2.5 Sn (ELI) } & \text { SQUARE BAR } & \text { CONDITION }\end{array}$

SPECIFICATIONS RB0170-079 DIRECTION LONGITUDINAL

PROPERTY ROTATING BEAM FATIGUE LIFE Q RT

\begin{tabular}{|c|c|c|c|c|c|c|c|c|c|c|}
\hline \multirow[b]{2}{*}{$\begin{array}{c}\text { STRESS } \\
\text { KSI }\end{array}$} & \multicolumn{6}{|c|}{ LOG OF CYCLES } & \multicolumn{2}{|c|}{ NO. OF CYCLES $\times 10^{3}$} & \multirow[b]{2}{*}{$\begin{array}{l}\text { DATA } \\
\text { CATEGORY }\end{array}$} & \multirow[b]{2}{*}{$\begin{array}{c}\text { SOURCE } \\
\text { REFERENCE }\end{array}$} \\
\hline & MEAN & $\begin{array}{c}\text { STANDARD } \\
\text { ERROR } \\
\text { OF } \\
\text { ESTIMATE } \\
\text { Se } \\
\end{array}$ & $\begin{array}{l}\text { EFFECTIVE } \\
\text { SAMPLE } \\
\text { SIZE }\end{array}$ & $\begin{array}{c}\text { DEGREES } \\
\text { OF } \\
\text { FREEDOM } \\
\text { FOR } \\
\text { se } \\
\end{array}$ & $k$ & $\begin{array}{l}\text { LOWER } \\
99 / 95 \\
\text { LIMIT }\end{array}$ & MEAN & $\begin{array}{c}\text { DESIGN } \\
\text { ALLOWABLE }\end{array}$ & & \\
\hline 100 & 4.25 & .242 & 11.4 & 21 & 3.31 & 3.45 & 18 & 3 & c & 1 \\
\hline 95 & 4.53 & .268 & 11.3 & $2]$ & 3.31 & 3.62 & 34 & 4 & C & 1 \\
\hline 90 & 4.82 & .304 & 11.3 & 21 & 3.32 & 3.81 & 66 & 7 & c & 1 \\
\hline 85 & 5.17 & .344 & 11.2 & 21 & 3.32 & 4.03 & 147 & 11 & c & 1 \\
\hline 80 & 5.56 & .392 & 11.0 & 21 & 3.32 & 4.26 & 367 & 18 & c & 1 \\
\hline 75 & 6.03 & .451 & 10.8 & 21 & 3.32 & 4.53 & 1069 & 34 & c & 1 \\
\hline
\end{tabular}


DESIGN DATA

MATERIAL

$T i-5 A 1-2.5 \mathrm{Sn}$ (ELI)

FORM

SQUARE BAR

CONDITION

ANNEALED

SPECIFICATIONS

RB 0170-079

DIRECTION

LONGITUDINAL

PROPERTY

ROTATING BEAM FATIGUE STRENGTH @ RT

NO. OF CYCLES

$\frac{M E A N}{X}$

(KSI)

\begin{tabular}{rrrrrr}
\hline $10^{5}$ & 87.4 & 4.8 & 69.0 & $c$ & 1 \\
$3.16 \times 10^{4}$ & 95.5 & 4.9 & 76.0 & $c$ & 1 \\
$10^{4}$ & 104.0 & 4.6 & 85.6 & $c$ & 1 \\
\hline
\end{tabular}

CATEGORY

SOURCE

REFERENCE
DESIGN DATA 


\section{TEST MATERIAL}

Axial Load $(R=0)$ and Rotating Beam $(R=-1)$ Fatigue testing were conducted on Ti-5Al-2.5Sn ELI bar stock as described in Reference (1). The axial load test was conducted at three temperatures, $-423^{\circ} \mathrm{F},-320^{\circ} \mathrm{F}$ and $70^{\circ} \mathrm{F}$ at a frequency of $1725 \mathrm{CPM}$. The rotating beam test was conducted at $-320^{\circ} \mathrm{F}$ and $70^{\circ} \mathrm{F}$, at a frequency of 10,000 CPM. Stress levels were selected so that failure would generally occur between $10^{4}$ and $10^{7}$ cycles. A total of 30 axial $10 a d$ and 26 rotating beams specimens were tested. The number of replications per stress level ranged from 1 to 4 .

\section{DATA ANALYSIS}

The data were analyzed by the method of multiple regression, in which the two independent variables were stress and temperature, and the dependent variable was the $\log$ of cycle life. The axial load and rotating beam tests were, of course, analyzed separately, but the temperatures were combined within each test type.

The Computer Program MULEFAT on the GE time-sharing computer was used to select regression models, to determine the coefficients of the associated regression equation, the standard error of estimate and the predicted cycle life at each stress level. The regression models were:

1. For Axial Load Test $Y=A+B X_{1}^{2}+C / X_{2}^{2}+D\left(X_{2} / X_{1}\right)$ where $Y=\log$ of cycle life, $X_{1}=$ temperature, ${ }^{\circ} \mathrm{F}, X_{2}=$ stress, KSI, and $A, B, C$ and $D$ are regression coefficients $(A=2.001$, $\left.B=3.635 \times 10^{-6}, C=73916.6, D--0.0112\right)$. The coefficient of determination was 0.921 , indicating a good fit of the model to the data. 


\section{For Rotating Beam Test}

$$
1 / Y=A+B X_{1}+C X_{2}
$$

where the variables are defined as above except $x_{1}=$ temp, ${ }^{\circ} \mathrm{R}$. $\left(A=-.261, B=-.002768, C=4104 \times 10^{-4}\right)$

The coefficient of determination was only .642 , indicated a poor fit to the data. However, the poor fit is due to high variability among replicates rather than to the inadequacy of the model.

The computer program REGLIM was used to compute the 99/95 limits for $\log$ cycle life and the effective sample size at each stress level. This program is based on a matrix method given in Reference (2). Because the regression equation for the Rotating Beam Test is in reciprocal form with respect to the dependent variable $(1 / Y)$, the standard error of estimate for $Y$ differs at the various stress levels. The means and lower limit of cycle life are shown both in logarithmic form and converted back to numbers of cycles. The low design allowable stress for Rotating Beam is a consequence of the high standard error which, in turn, is a result of the poor reproducibility among rep1icates.

The means and lower limits were plotted versus stress for each temperature. From these plots, mean stress and allowable stress corresponding to various cycle lives were determined. The standard deviations of these estimates were calculated by a method based on para. 5.3.5 of Reference (3).

The data have been categorized as " $C$ " because lots are not identified and lot-to-lot variation is not considered. 
3. REFERENCES:

(1) Rocketdyne Report R-7564, "Fatigue Properties of Sheet, Bar and Cast Metallic Materials for Cryogenic Applications," dated 30 August 1968.

(2) Draper and Smith, "Applied Regression Analysis," Wiley, 1966, p. 122.

(3) NERVA Program Procedure R101-NRP-502, "Sampling for Fatigue Test". 


\section{AEROJET NUCLEAR SYSTEMS COMPANY}

MATERIALS DATA RELEASE

INTERNAL DISTRIBUTIOI

C. K. Leeper

G. F. Mader

J. H. Ramsthaler (2)

L. Shenfil

P. P. Ventura

F. E. Porter

W. E. Campbel1

N. A. Edlebeck (2.

L. A. Shurley (2)

L. D. Johnson (2)

A. D. Cornel1

U. A. Pineda (2)

K. Sato

E. A. Warman

T. A. Redfield

0.J. Demuth

H. Derow

C. E. Dixon

P. P. Dessau

M. S. Lev 
MATERIAL Ti-5Al-2.5 Sn (ELI)

ANSC

MATERIALS DATA RELEASE

CONTENTS

\begin{tabular}{|c|c|c|c|c|c|}
\hline MATERIAL & FORM & CONDITION & PROPERTY & $\begin{array}{c}\text { DATA } \\
\text { CATEGORY } \\
\end{array}$ & PAGE \\
\hline$T i-5 A 1-2.5 \mathrm{Sn}$ (ELI) & Bar & Annealed & Axial Load Fatigue Life $0-423^{\circ} \mathrm{F}$ & $c$ & 2 \\
\hline$"$ & $"$ & $"$ & Axial Load Fatigue Strength $0-423^{\circ} \mathrm{F}$ & C & 3 \\
\hline$"$ & $"$ & $"$ & Axial Load Fatigue Life a $-320^{\circ} \mathrm{F}$ & C & 4 \\
\hline$"$ & $"$ & $"$ & Axial Load Fatigue Strength $0-320^{\circ} \mathrm{F}$ & $c$ & 5 \\
\hline$"$ & $"$ & $"$ & Axial Load Fatigue Life a RT & c & 6 \\
\hline$"$ & $"$ & $"$ & Axial Load Fatigue Strength O RT & C & 7 \\
\hline$"$ & Square Bar & & Rotating Beam Fatigue Life a $-320^{\circ} \mathrm{F}$ & c & 8 \\
\hline$"$ & $"$ & $"$ & Rot. Roam Fatigue Strength A $-320^{\circ}$ F & $c$ & 9 \\
\hline " & " & $"$ & Rotating Beam Fatigue Life o RT & C & 10 \\
\hline " & $"$ & $"$ & Rotating Beam Fatigue Strength a RT & c & 11 \\
\hline
\end{tabular}

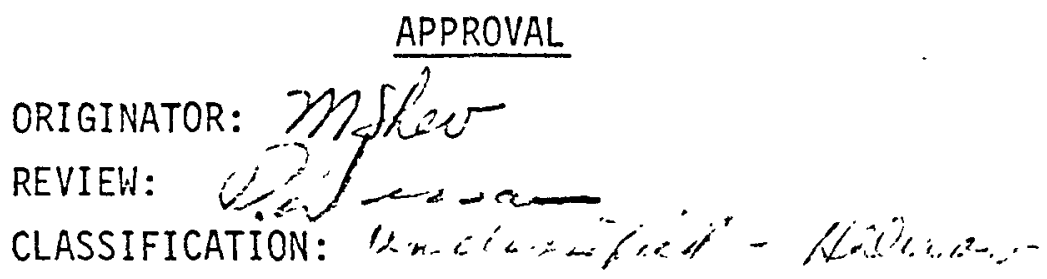




$\begin{array}{lllll}\text { MATERIAL } & \text { Ti-5AI-2.5 Sn (ELI) } & \text { FORM } & \text { BAR } & \text { CONDITION } \\ \text { SPECIFICATIONS } & \text { RB } 0170-79 & \text { DIRECTION } & \underline{\text { AXIAL }}\end{array}$

PROPERTY AXIAL LOAD FATIGUE LIFE $0-423$

\begin{tabular}{|c|c|c|c|c|c|c|c|c|c|c|}
\hline \multirow[b]{2}{*}{$\begin{array}{c}\text { STRESS } \\
\text { KSI }\end{array}$} & \multicolumn{4}{|c|}{ LOG OF CYCLES } & & & \multicolumn{2}{|c|}{ NO. OF CYCLES $\times 10^{3}$} & \multirow[b]{2}{*}{$\begin{array}{l}\text { DATA } \\
\text { CATEGORY }\end{array}$} & \multirow[b]{2}{*}{$\begin{array}{l}\text { SOURCE } \\
\text { REFERENCE }\end{array}$} \\
\hline & MEAN & $\begin{array}{l}\text { STANDARD } \\
\text { ERROR } \\
\text { OF } \\
\text { ESTIMATE } \\
\text { se }\end{array}$ & $\begin{array}{c}\text { EFFECTIVE } \\
\text { SAMPLE } \\
\text { SIZE } \\
m \\
\end{array}$ & $\begin{array}{c}\text { DEGREES } \\
\text { OF } \\
\text { FREEDOM } \\
\text { FOR } \\
\text { se }\end{array}$ & $k$ & $\begin{array}{l}\text { LOWER } \\
99 / 95 \\
\text { LIMIT }\end{array}$ & MEAN & $\begin{array}{l}\text { DESIGN } \\
\text { ALLOWABLE }\end{array}$ & & \\
\hline 200 & 4.26 & 0.231 & 10.3 & 26 & 3.24 & 3.51 & 18 & 3 & C & 1 \\
\hline 190 & 4.35 & 0.231 & 10.3 & 26 & 3.24 & 3.61 & 23 & 4 & C & 1 \\
\hline 180 & 4.48 & 0.231 & 10.4 & 26 & 3.24 & 3.74 & 30 & 5 & C & 1 \\
\hline 170 & 4.67 & 0.231 & 10.5 & 26 & 3.24 & 3.93 & 47 & 8 & C & 1 \\
\hline 160 & 4.92 & 0.231 & 10.5 & 26 & 3.24 & 4.77 & 82 & 15 & C & 1 \\
\hline 150 & 5.23 & 0.231 & 10.5 & 26 & 3.24 & 4.48 & 171 & 30 & C & 7 \\
\hline 140 & 5.64 & 0.237 & 10.3 & 26 & 3.24 & 4.89 & . 438 & 78 & C & 1 \\
\hline 130 & 6.17 & 0.231 & 10.3 & 26 & 3.24 & 5.42 & 1486 & 265 & C & 1 \\
\hline
\end{tabular}


DESIGN DATA

DRM: $\quad 1.05$

DATE : $4-27-71$

Page 4 of 14

MATERIAL

$T i-5 A 7-2.5 \mathrm{Sn}$ (ELI)

FORM

$\underline{B A R}$

CONDITION

ANNEALED

SPECIFICATIONS

RB $\quad 0770-079$

DIRECTION

AXIAL

PROPERTY

AXIAL LOAD FATIGUE LIFE @ $-320^{\circ} \mathrm{F}$

\begin{tabular}{|c|c|c|c|c|c|c|c|c|c|c|}
\hline \multirow[b]{2}{*}{$\begin{array}{l}\text { STRESS } \\
\text { (KSI) }\end{array}$} & \multicolumn{5}{|c|}{ LOG OF CYCLES } & & \multicolumn{2}{|c|}{ NO. OF CYCLES $\times 10^{3}$} & \multirow[b]{2}{*}{$\begin{array}{l}\text { DATA } \\
\text { CATEGORY }\end{array}$} & \multirow[b]{2}{*}{$\begin{array}{l}\text { SOURCE } \\
\text { REFERENCE }\end{array}$} \\
\hline & MEAN & $\begin{array}{l}\text { STANDARD } \\
\text { ERROR } \\
\text { OF } \\
\text { ESTIMATE } \\
\text { Se } \\
\end{array}$ & $\begin{array}{c}\text { EFFECTIVE } \\
\text { SAMPLE } \\
\text { SIZE } \\
m\end{array}$ & $\begin{array}{l}\text { DEGREES } \\
\text { OF } \\
\text { FREEDOM } \\
\text { FOR } \\
\text { se } \\
\end{array}$ & $k$ & $\begin{array}{l}\text { LOWER } \\
99 / 95 \\
\text { LIMIT }\end{array}$ & MEAN & $\begin{array}{c}\text { DESIGN } \\
\text { ALLOWABLE }\end{array}$ & & \\
\hline 190 & 4.94 & .237 & 17.4 & 26 & 3.16 & 4.21 & 87 & 16 & $c$ & 1 \\
\hline 180 & 5.04 & .231 & 17.7 & 26 & 3.16 & 4.31 & 111 & 21 & C & 1 \\
\hline 170 & 5.20 & .231 & 18.2 & 26 & 3.16 & 4.47 & 158 & 29 & $C$ & 1 \\
\hline 160 & 5.41 & .231 & 18.5 & 26 & 3.15 & 4.68 & 258 & 48 & C & 1 \\
\hline 150 & 5.70 & .231 & 39.0 & 26 & 3.75 & 4.97 & 503 & 94 & C & 1 \\
\hline 140 & 6.09 & .231 & 19.2 & 26 & 3.15 & 5.34 & 759 & 142 & C & 1 \\
\hline 130 & 6.59 & .231 & 19.4 & 26 & 3.15 & 5.87 & 3926 & 735 & C & 1 \\
\hline 120 & 7.24 & .231 & 19.6 & 26 & 3.15 & 6.54 & 17380 & 3467 & C & 1 \\
\hline
\end{tabular}


DESIGN DATA

\begin{tabular}{lllll} 
MATERIAL & Ti-5AT-2.5 Sn (ELI) & FORM & BAR & CONDITION \\
\cline { 2 - 3 } & $\underline{\text { RBO } 170-079}$ & DIRECTION & $\underline{\text { AXIAL }}$
\end{tabular}

PROPERTY AXIAL FATIGUE STRENGTH $0-320^{\circ} \mathrm{F}$

\begin{tabular}{|c|c|c|c|c|c|}
\hline NO. OF CYCLES & $\begin{array}{c}\bar{X} \\
(K S I)\end{array}$ & $\mathbf{s}$ & $\begin{array}{c}\text { DESIGN } \\
\text { ALLOWABLE } \\
(\mathrm{KSI})\end{array}$ & $\begin{array}{c}\text { DATA } \\
\text { CATEGORY }\end{array}$ & $\begin{array}{c}\text { SOURCE } \\
\text { REFERENCE }\end{array}$ \\
\hline $3.16 \times 10^{6}$ & 132 & 4.28 & 120.5 & C & 1 \\
\hline $3.16 \times 10^{5}$ & 156 & 8.70 & 136.5 & C & 1 \\
\hline $10^{5}$ & 184 & 15.10 & 149 & C & 1 \\
\hline
\end{tabular}


DESIGN DATA

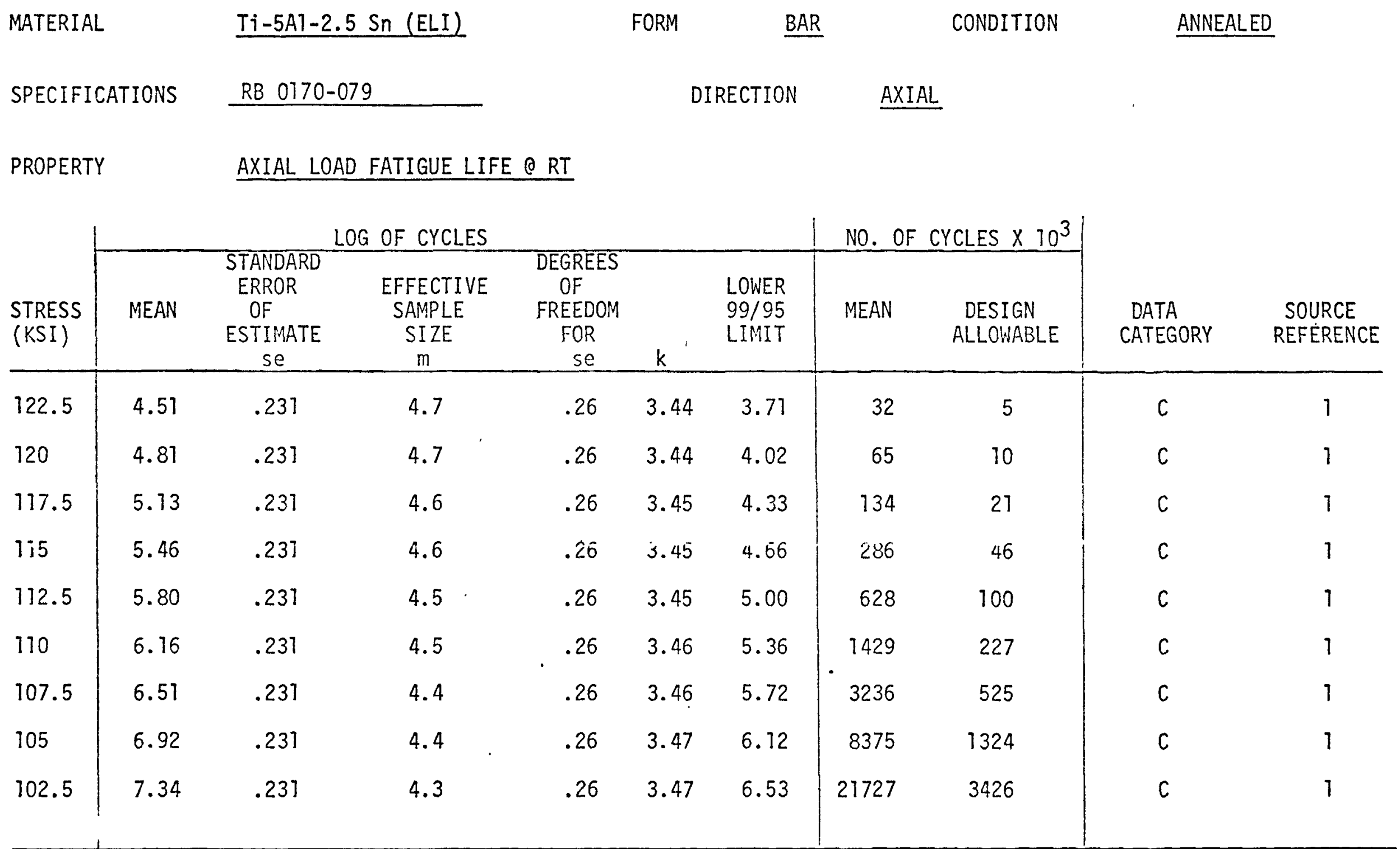


DESIGN DATA

$\begin{array}{lcccc}\text { MATERIAL } & \text { Ti-5AT-2.5 Sn }(\dot{E} L I) & \text { FORM } & \text { CONDITION } & \text { ANNEALED } \\ \text { SPECIFICATIONS } & \underline{\text { RBOT70-079 }} & \text { DIRECTION } & \underline{\text { AXIAL }}\end{array}$

PROPERTY AXIAL FATIGUE STRENGTH O RT

\begin{tabular}{|c|c|c|c|c|c|}
\hline NO. OF CYCLES & $\begin{array}{c}\bar{X} \\
(K S I) \\
\end{array}$ & $\mathrm{s}$ & $\begin{array}{c}\text { DESIGN } \\
\text { ALLOWABLE } \\
(K S I)\end{array}$ & $\begin{array}{l}\text { DATA } \\
\text { CATEGORY }\end{array}$ & $\begin{array}{l}\text { SOURCE } \\
\text { REFERENCE }\end{array}$ \\
\hline $3.16 \times 10^{6}$ & 107.6 & 1.60 & 102.7 & C & 1 \\
\hline $3.16 \times 10^{5}$ & 114.7 & 1.72 & 109.0 & C & 1 \\
\hline $10^{5}$ & $\cdot 118.4$ & 1.75 & 112.5 & C & 1 \\
\hline $3.16 \times 10^{4}$ & 122.5 & 1.84 & 116.2 & C & 1 \\
\hline
\end{tabular}


DESIGN DATA

$\begin{array}{lllll}\text { MATERIAL } & \text { Ti-5AT-2.5 Sn (ELI) } & \text { FORM } & \text { SQUARE BAR } & \text { CONDITION } \\ \text { SPECIFICATIONS } & \text { RBOT70-079 } & \text { DIRECTION } & \text { LONGITUDINAL }\end{array}$

PROPERTY ROTATING BEAM FATIGUE LIFE $0-320^{\circ} \mathrm{F}$

\begin{tabular}{|c|c|c|c|c|c|c|c|c|c|c|}
\hline \multirow[b]{2}{*}{$\begin{array}{l}\text { STRESS } \\
\text { (KSI) }\end{array}$} & \multicolumn{6}{|c|}{ LOG OF CYCLES } & \multicolumn{2}{|c|}{ NO. OF CYCLES $\times 10^{3}$} & \multirow[b]{2}{*}{$\begin{array}{l}\text { DATA } \\
\text { CATEGORY }\end{array}$} & \multirow[b]{2}{*}{$\begin{array}{l}\text { SOURCE } \\
\text { REFERENCE }\end{array}$} \\
\hline & MEAN & $\begin{array}{c}\text { STANDARD } \\
\text { ERROR } \\
\text { OF } \\
\text { ESTIMATE } \\
\text { Se }\end{array}$ & $\begin{array}{l}\text { EFFECTIVE } \\
\text { SAMPLE } \\
\text { SIZE }\end{array}$ & $\begin{array}{c}\text { DEGREES } \\
\text { OF } \\
\text { FREEDOM } \\
\text { FOR } \\
\text { se } \\
\end{array}$ & $k$ & $\begin{array}{l}\text { LOWER } \\
99 / 95 \\
\text { LIMIT }\end{array}$ & MEAN & $\begin{array}{c}\text { DESIGN } \\
\text { ALLOWABLE }\end{array}$ & & \\
\hline 150 & 4.73 & 0.290 & 8.5 & 21 & 3.37 & 3.75 & 54 & 6 & $C$ & 1 \\
\hline 145 & 5.08 & 0.330 & 9.2 & 21 & 3.36 & 3.95 & 120 & 9 & C & 1 \\
\hline 140 & 5.44 & 0.376 & 9.7 & 21 & 3.35 & 4.19 & 278 & 15 & C & 1 \\
\hline 135 & 5.89 & 0.432 & 10.2 & 21 & 3.33 & 4.45 & 773 & 28 & C & 1 \\
\hline 130 & 6.41 & 0.502 & 10.8 & 21 & 3.32 & 4.74 & 2574 & 55 & C & 1 \\
\hline
\end{tabular}


DESIGN DATA

\begin{tabular}{|c|c|c|c|c|c|}
\hline MATERIAL & $T i-5 A]-2.5 \mathrm{Sn}$ (ELI) & FORM & RE BAR & CONDITION & ANNEALED \\
\hline SPECIFICATIONS: & RB $\quad 0170-079$ & DIRECTION & LONGI & & \\
\hline PROPERTY & \multicolumn{5}{|c|}{ ROTATING BEAM FATIGUE STRENGTH $@-320$} \\
\hline
\end{tabular}

\begin{tabular}{|c|c|c|c|c|c|}
\hline NO. OF CYCLES & $\begin{array}{c}\text { MEAN } \\
\bar{X} \\
(K S I)\end{array}$ & $\mathrm{s}$ & $\begin{array}{l}\text { DESIGN } \\
\text { ALLOWABLE } \\
(\text { KSI) }\end{array}$ & $\begin{array}{c}\text { DATA } \\
\text { CATEGORY }\end{array}$ & $\begin{array}{c}\text { SOURCE } \\
\text { REFERENCE }\end{array}$ \\
\hline $10^{5}$ & 146.0 & 5.0 & 126.8 & $C$ & 1 \\
\hline $3.16 \times 10^{4}$ & 154.0 & 6.1 & 134.0 & C & 1 \\
\hline
\end{tabular}


DRM: $\leadsto .05$

DATE: $27-71$

Page 10 of 14

DESIGN DATA

\begin{tabular}{|c|c|c|c|c|c|c|c|c|c|c|}
\hline MATERIAL & & $T i-5 A T-2.5$ & $(E L I)$ & FO & & SQUAR & BAR & CONDITION & ANNEA & \\
\hline SPECIFIC & TIONS & RB0170-079 & & & RECTION & & ITUDINA & & & \\
\hline PROPERTY & & ROTATING BE & 1 FATIGUE LI & Q RT & & & & & & \\
\hline & & & LOG OF CYCL & & & & NO. 0 & YYCLES $\times 10^{3}$ & & \\
\hline $\begin{array}{l}\text { STRESS } \\
\text { KSI }\end{array}$ & MEAN & $\begin{array}{l}\text { STANDARD } \\
\text { ERROR } \\
\text { OF } \\
\text { ESTIMATE } \\
\text { Se } \\
\end{array}$ & $\begin{array}{l}\text { EFFECTIVE } \\
\text { SAMPLE } \\
\text { SIZE }\end{array}$ & $\begin{array}{c}\text { DEGREES } \\
\text { OF } \\
\text { FREEDOM } \\
\text { FOR } \\
\text { se } \\
\end{array}$ & k & $\begin{array}{l}\text { LOWER } \\
\text { 99/95 } \\
\text { LIMIT }\end{array}$ & MEAN & $\begin{array}{c}\text { DESIGN } \\
\text { ALLOWABLE }\end{array}$ & $\begin{array}{l}\text { DATA } \\
\text { CATEGORY }\end{array}$ & $\begin{array}{l}\text { SOURCE } \\
\text { REFERENCE }\end{array}$ \\
\hline 100 & 4.25 & .242 & 11.4 & 27 & 3.37 & 3.45 & 18 & 3 & C & 1 \\
\hline 95 & 4.53 & .268 & 11.3 & 21 & 3.31 & 3.62 & 34 & 4 & C & 1 \\
\hline 90 & 4.82 & .304 & 11.3 & 21 & 3.32 & 3.81 & 66 & 7 & C & 1 \\
\hline 85 & 5.77 & .344 & 11.2 & 21 & 3.32 & 4.03 & 147 & 11 & C & 1 \\
\hline 80 & 5.56 & .392 & 11.0 & 27 & 3.32 & 4.26 & 367 & 18 & C & 1 \\
\hline 75 & 6.03 & .451 & 10.8 & 27 & 3.32 & 4.53 & 1069 & 34 & c & 1 \\
\hline
\end{tabular}


DESIGN DATA

$\begin{array}{lclll}\text { MATERIAL } & \text { Ti-5AI-2.5 Sn (ELI) } & \text { FORM } & \text { SQUARE BAR } & \text { CONDITION } \\ \text { SPECIFICATIONS } & \text { RB } 0170-079 & \text { DIRECTION } & \text { LONGITUDINAL }\end{array}$

PROPERTY ROTATING BEAM FATIGUE STRENGTH O RT

\begin{tabular}{|c|c|c|c|c|c|}
\hline NO. OF CYCLES & $\begin{array}{l}\text { MEAN } \\
\bar{X} \\
(K S I)\end{array}$ & $S$ & $\begin{array}{c}\text { DESIGN } \\
\text { ALLOWABLE }\end{array}$ & $\begin{array}{c}\text { DATA } \\
\text { CATEGORY }\end{array}$ & $\begin{array}{l}\text { SOURCE } \\
\text { REFERENCE }\end{array}$ \\
\hline $10^{5}$ & 87.4 & 4.8 & 69.0 & C & 1 \\
\hline $3.16 \times 10^{4}$ & 95.5 & 4.9 & 76.0 & C & 1 \\
\hline $10^{4}$ & 104.0 & 4.6 & 85.6 & C & 1 \\
\hline
\end{tabular}




\section{TEST MATEPIAL}

Axial Load $(R=0)$ and Rotating Beam $(R=-1)$ Fatigue testing were conducted on Ti-5AT-2.5Sn ELI bar stock as descrited in Reference (1). The axial load test was confucted at three temperatures, $-423^{\circ} \mathrm{F},-320^{\circ} \mathrm{F}$ and $70^{\circ} \mathrm{F}$ at a frequency of $1725 \mathrm{CPM}$. The rotating beam test was conducted at $-320^{\circ} \mathrm{F}$ and $70^{\circ} \mathrm{F}$, at a frequency of $10,000 \mathrm{CPM}$. Stress levels were selected so that failure woutd generally occur between $10^{4}$ and $10^{7}$ cycles. A total of 30 axial 1 oad and 26 rotating beams spæcimens were tested. The number of replications per stres: level ranged from 1 to 4 .

\section{DATA ANALISIS}

The data were analyzed by the method of multiple regression, in which the two independent variables were stress and temperature, and the dependent var-able was the log of cy:le life. The axial load and rotating beam tests were, of course, analyzed separately, but the temperatures were combined within each test typ..

The Compuser Program MULEFAT on the GE time-sharing computer was use'. to select regression models, to determine the coefficients of the associated regression equation, the standard error of estimate and the predicted cycle life at each stress leve1. The regression modeis were:

1. For Axial Load Test

$$
Y=A+B X_{1}^{2}+C / X_{2}^{2}+D\left(X_{2} / X_{1}\right)
$$

where $Y=\log$ of cycle life, $X_{1}=$ temperature, ${ }^{\circ} \mathrm{F}, X_{2}=$ stress, KSI, and $A, B, C$ and $D$ are regression coefficients $(A=2.001$, $\left.B=3.635 \times 10^{-6}, C=73916.6, D--0.0112\right)$. The coefficient of determination was 0.921 , indicating a good fit of the model to the data. 
2. For Rolating Beam Test

$$
1 / Y=A+B X_{1}+C X_{2}
$$

where the variables are defined as a'sove except $X_{1}=$ temp, ${ }^{\circ} \mathrm{R}$. $\left(A=-.261, B=-.002768, C=4104 \times 10^{-4}\right)$

The coefficient of determination was only .642 , indicated a poor fit to the data. However, the poor fit is due to high variability among replicates rather than to the inadequacy of the model.

The computer program REGLIM was used to compute the 99/95 limits for log cycle life and the effective sample size at each stress level. This program is based on a matrix method given in Reference (2). Because the regression equaticn for the Rotating Beam Test is in reciprocal form with respect to the dependent variable $(1 / Y)$, the standard error of estimate for $Y$ differs at the various stress levels. The means and lower limit of cycle life are shown both in logarithmic form and converted back to numbers of cycles. The low design allowable stress for Rotating Beam is a consequence of the high standard error which, in turn, is a result of the poor reproducibility among replicates.

The means and lower limits were plotted versus stress for each temperature. From these plots, mean stress and allowable stress corresponding to various cycle lives were determined. The standard deviations of these estimates were calculated by a method based on para. 5.3.5 of Reference (3).

The data have been categorized as " $C$ " because lots are not identified and lot-to-lot variation is not considered. 
DRN1: 04.05

DATE : $4-27-71$

Page 14 of 14

\section{REFERENCES:}

(1) Rocketdyne Report R-7564, "Fatigue Properties of Sheet, Bar and Cast Metallic Materials for Cryogenic Applications," dated 30 August 1968.

(2) Draper and Smith, "Applied Regression Analysis," Wiley, 1966, p. 122.

(3) NERVA Program Procedure R101-NRP-5C2, "Sampling for Fatigue Test". 
MATERIAL

A-286

DRM: $\quad 05.04$

DATE: $4-21-71$

PAGE: 1 of 10

\section{ANSC \\ MATERIALS DATA RELEASE}

\begin{tabular}{|c|c|c|c|c|c|}
\hline MATERIAL & FORM & CONDITION & PROPERTY & ATEGORY & PAGE \\
\hline$A-286$ & Sheet & Annealed \& Aged & F]exura] Fatigue Life $0-423^{\circ} \mathrm{F}$ & $c$ & 2 \\
\hline$"$ & $"$ & $"$ & Flexural Fatigue Strength o $-423^{\circ} \mathrm{F}$ & $c$ & 3 \\
\hline$"$ & $"$ & $"$ & Flexural Fatigue Life $0-320^{\circ} \mathrm{F}$ & $c$ & 4 \\
\hline$"$ & $"$ & $"$ & Flexural Fatigue Strength a $-320^{\circ} \mathrm{F}$ & $c$ & 5 \\
\hline$"$ & $"$ & $"$ & Flexural Fatigue Life a RT & $c$ & 6 \\
\hline$"$ & $"$ & $"$ & Flexural Fatigue Strength @ RT & c & 7 \\
\hline
\end{tabular}

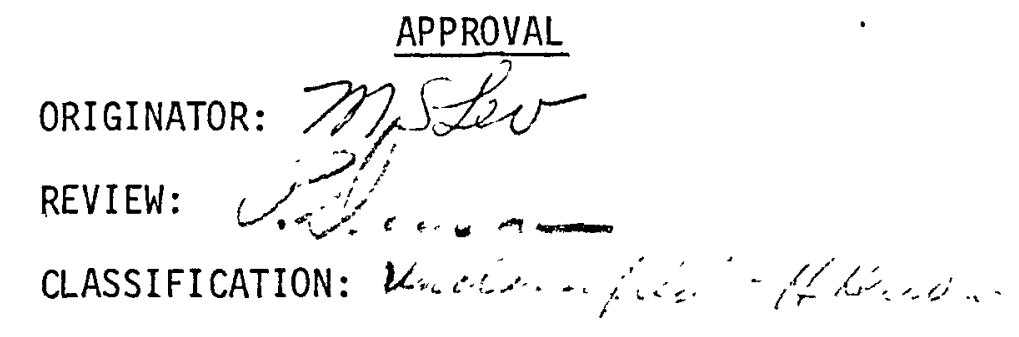


DESIGN DATA

MATERIAL

A-286

FORM

SHEET

SPECIFICATIONS AMS 5525

FLEXURAL FATIGUE LIFE AT $-423^{\circ} \mathrm{F}$

PROPERTY
DRM: $\quad$ US.04

DATE: $4-27-71$

PAGE: 2 of 10

CONDITION

ANNEALED \& AGED

FLEXURE NORMAL TO DIRECTION OF ROLLING

\begin{tabular}{|c|c|c|c|c|c|c|c|c|c|c|c|}
\hline & $\begin{array}{c}\text { STRESS } \\
(\mathrm{KSI})\end{array}$ & $\frac{L O G}{\text { MEAN }}$ & $\begin{array}{c}\text { OF } \quad \text { C } \\
\text { STANDARD } \\
\text { ERROR OF } \\
\text { ESTIMATE } \\
\text { se }\end{array}$ & $\begin{array}{l}C L E S \\
\text { EFFECTIVE } \\
\text { SAMPLE } \\
\text { SIZE }\end{array}$ & $\begin{array}{c}\text { DEGREES } \\
\text { OF } \\
\text { FREEDOM } \\
\text { FOR } \mathrm{se}_{\mathrm{e}}\end{array}$ & $k$ & $\begin{array}{l}\text { LOWER } \\
99 / 95 \\
\text { LIMIT }\end{array}$ & \multicolumn{2}{|c|}{$\begin{array}{r}\text { NUMBER OF CYCLES } \\
\times 10^{3} \\
\end{array}$} & $\begin{array}{l}\text { DATA } \\
\text { CATEGORY }\end{array}$ & $\begin{array}{l}\text { SOURCE } \\
\text { REFERENCE }\end{array}$ \\
\hline \multirow[t]{2}{*}{ A. } & SURFACE & FINISH $=$ & 10 RMS & & & & & & & & \\
\hline & $\begin{array}{l}132.5 \\
130 \\
125 \\
120 \\
115 \\
112.5\end{array}$ & $\begin{array}{l}4.44 \\
4.64 \\
5.05 \\
5.46 \\
5.88 \\
6.08\end{array}$ & $\begin{array}{l}.106 \\
.106 \\
.106 \\
.106 \\
.106 \\
.106\end{array}$ & $\begin{array}{l}6.2 \\
6.5 \\
7.0 \\
7.7 \\
8.3 \\
8.8\end{array}$ & $\begin{array}{l}21 \\
21 \\
21 \\
21 \\
21 \\
21\end{array}$ & $\begin{array}{l}3.45 \\
3.44 \\
3.41 \\
3.39 \\
3.38 \\
3.37\end{array}$ & $\begin{array}{l}4.07 \\
4.27 \\
4.69 \\
5.10 \\
5.52 \\
5.73\end{array}$ & $\begin{array}{r}27 \\
43 \\
112 \\
290 \\
752 \\
1211\end{array}$ & $\begin{array}{r}12 \\
19 \\
49 \\
127 \\
330 \\
532\end{array}$ & $\begin{array}{l}c \\
c \\
c \\
c \\
c \\
c\end{array}$ & $\begin{array}{l}1 \\
1 \\
1 \\
1 \\
1 \\
1\end{array}$ \\
\hline \multirow[t]{2}{*}{ B. } & SURFACE & FINISH $=$ & 72 RMS & & & & & & & & \\
\hline & $\begin{array}{r}130 \\
125 \\
120 \\
115 \\
110 \\
105 \\
100 \\
95 \\
90 \\
85\end{array}$ & $\begin{array}{l}4.36 \\
4.54 \\
4.71 \\
4.88 \\
5.06 \\
5.23 \\
5.40 \\
5.57 \\
5.75 \\
5.92\end{array}$ & $\begin{array}{l}.106 \\
.106 \\
.106 \\
.106 \\
.106 \\
.106 \\
.106 \\
.106 \\
.106 \\
.106\end{array}$ & $\begin{array}{l}9.4 \\
9.3 \\
9.2 \\
9.0 \\
8.8 \\
8.5 \\
8.0 \\
7.7 \\
7.3 \\
7.0\end{array}$ & $\begin{array}{l}27 \\
27 \\
2] \\
21 \\
27 \\
27 \\
27 \\
27 \\
21 \\
21\end{array}$ & $\begin{array}{l}3.35 \\
3.35 \\
3.36 \\
3.36 \\
3.37 \\
3.37 \\
3.38 \\
3.39 \\
3.40 \\
3.42\end{array}$ & $\begin{array}{l}4.01 \\
4.18 \\
4.35 \\
4.53 \\
4.70 \\
4.87 \\
5.04 \\
5.21 \\
5.39 \\
5.56\end{array}$ & $\begin{array}{r}23 \\
34 \\
51 \\
76 \\
114 \\
169 \\
252 \\
375 \\
558 \\
832\end{array}$ & $\begin{array}{r}10 \\
15 \\
23 \\
34 \\
50 \\
74 \\
110 \\
164 \\
243 \\
361\end{array}$ & $\begin{array}{l}c \\
c \\
c \\
c \\
c \\
c \\
c \\
c \\
c \\
c\end{array}$ & $\begin{array}{l}1 \\
1 \\
1 \\
1 \\
1 \\
1 \\
1 \\
1 \\
1 \\
1\end{array}$ \\
\hline
\end{tabular}


DESIGN DATA

\begin{tabular}{|c|c|c|c|}
\hline MATERIAL & $A-286$ & SHEET & ANNEALED \& AGED \\
\hline SPECIFICATIONS & AMS 5525 & DIRECTION & FLEXURE NORMAL. TO DIRECTION OF ROLLING \\
\hline PROPERTY & ELEXURAL FAT & E STRENGTH $0-423$ & \\
\hline
\end{tabular}

\begin{tabular}{cccccc}
\hline NO. OF CYCLES & $\begin{array}{c}\bar{X} \\
(K S I)\end{array}$ & $k$ & $s$ & $\begin{array}{c}\text { DESIGN } \\
\text { ALLOWABLE } \\
(\text { KSI })\end{array}$ & $\begin{array}{c}\text { DATA } \\
\text { CATEGORY }\end{array}$ \\
\hline
\end{tabular}

A. SURFACE FINISH $=10$ RMS

$\begin{array}{rrrrrrr}10^{6} & 113.5 & 3.37 & 1.34 & 109.0 & c & 1 \\ * 3.16 \times 10^{5} & 119.6 & 3.39 & 1.33 & 115.1 & c & 1 \\ 10^{5} & 125.5 & 3.42 & 1.26 & 121.2 & c & 1 \\ 3.16 \times 10^{4} & 131.6 & 3.44 & 1.28 & 127.2 & c & 1 \\ 10^{4} & 137.6 & 3.47 & 1.27 & 133.2 & c & 1\end{array}$

B. SURFACE FINISH $=72$ RMS
$3.16 \times 10^{5}$
$10^{5}$
$3.16 \times 10^{4}$
$10^{4}$

100.0
113.0
126.0
140.0

3.38

2.96

90.0

c

103.0

c

116.0

130.0

c

c

* $3.16 \times 10^{5}=10^{5.5}=$ ANTILOG 5.5 
DESIGN DATA

MATERIAL

SPECIFICATIONS

PROPERTY
A-286

AMS 5525

FLEXURAL FATIGUE LIFE AT $-320^{\circ} \mathrm{F}$
DRM: 05.04

DATE: $4-27-71$ PAGE: 4 of 10 .

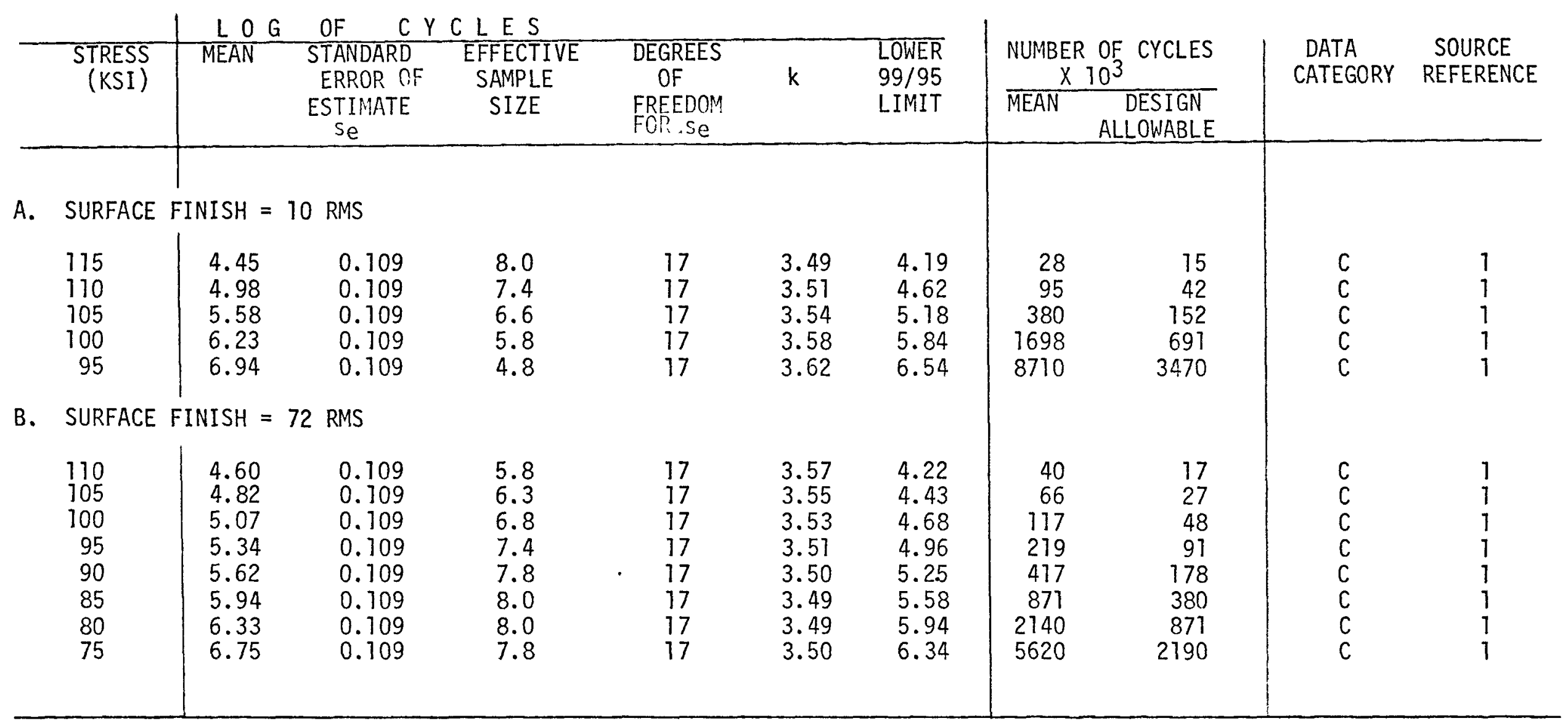


DESIGN DATA

\begin{tabular}{|c|c|c|c|c|}
\hline MATERIAL & $\underline{A-286}$ & SHEET & CONDITION & ANNEALED \& AGED \\
\hline SPECIFICATIONS & AMS 5525 & DIRECTION & FLEXURE NORMAL TO & DIRECTION OF ROLLING \\
\hline PROPERTY & FLEXURAL FAT] & STRENGTH @- 320 & & \\
\hline
\end{tabular}

FLEXURAL FATIGUE STRENGTH @ -320

NO. OF CYCLES

$\bar{X}$

$\bar{X}$

k s

DESIGN

ALLOWABLE

DATA

CATEGORY (KSI)

A. SURFACE FINISH $=10$ RMS

$\begin{array}{crrrrrr}10^{7} & 94.6 & 3.63 & 0.72 & 92.0 & c & 1 \\ \star 3.16 \times 10^{6} & 98.0 & 3.59 & 0.78 & 95.2 & c & 1 \\ 10^{6} & 101.7 & 3.56 & 0.81 & 98.8 & c & 1 \\ 3.16 \times 10^{5} & 105.7 & 3.53 & 0.91 & 102.5 & c & 1 \\ 10^{5} & 109.8 & 3.51 & 0.94 & 106.5 & c & 1 \\ 3.16 \times 10^{4} & 114.5 & 3.49 & 0.97 & 111.1 & c & 1 \\ 10^{4} & 119.8 & 3.47 & 1.12 & 115.9 & c & 1\end{array}$

B. SURFACE FINISH $=72$ RMS

$\begin{array}{crrrr}3.16 \times 10^{6} & 77.9 & 3.49 & 1.35 & 73.2 \\ 10^{6} & 84.2 & 3.49 & 1.43 & 79.2 \\ 3.16 \times 10^{5} & 92.1 & 3.50 & 1.74 & 86.0 \\ 10^{5} & 101.3 & 3.53 & 1.98 & 94.3 \\ 3.16 \times 10^{4} & 113.0 & 3.58 & 2.65 & 103.5\end{array}$

* $3.16 \times 10^{6}=10^{6.5}=$ ANTILOG 5.5 
DESIGN DATA

MATERIAL

A-286

FORM

SHEET

SPECIFICATIONS

PROPERTY

AMS 5525

\section{FLEXURAL FATIGUE LIFE @ RT}

DRIY: 05.04

DATE: $4-27-71$

PAGE: 6 of 10

CONDITION

ANNEALED \& AGED

FLEXURE NORMAL TO DIRECTION OF ROLLING

\begin{tabular}{|c|c|c|c|c|c|c|c|c|c|c|}
\hline $\begin{array}{c}\text { STRESS } \\
(\text { KSI })\end{array}$ & MEAN & $\begin{array}{l}\text { G OF } \\
\text { STANDARD } \\
\text { ERROR OF } \\
\text { ESTIMATE } \\
\text { se }\end{array}$ & $\begin{array}{l}Y C L E \text { Y S } \\
\text { EFFECTIVE } \\
\text { SAMPLE } \\
\text { SIZE }\end{array}$ & $\begin{array}{l}\text { DEGREES } \\
\text { OF } \\
\text { FREEDOM } \\
\text { FOR se }\end{array}$ & $\mathrm{k}$ & $\begin{array}{l}\text { LOWER } \\
99 / 95 \\
\text { LIMIT }\end{array}$ & \multicolumn{2}{|c|}{$\begin{array}{r}\text { NUMBER OF CYCLES } \\
\times 10^{3} \\
\text { MEAN } \\
\\
\text { ALLESIGN } \\
\text { ALOWABLE }\end{array}$} & $\begin{array}{l}\text { DATA } \\
\text { CATEGORY }\end{array}$ & $\begin{array}{c}\text { SOURCE } \\
\text { REFERENCE }\end{array}$ \\
\hline \multicolumn{11}{|c|}{ A. SURFACE FINISH $=10$ RMS } \\
\hline $\begin{array}{l}95 \\
90 \\
85 \\
80 \\
75 \\
70 \\
65 \\
62.5\end{array}$ & $\begin{array}{l}4.35 \\
4.63 \\
4.96 \\
5.31 \\
5.73 \\
6.23 \\
6.87 \\
7.15\end{array}$ & $\begin{array}{l}.079 \\
.089 \\
.100 \\
.116 \\
.135 \\
.158 \\
.188 \\
.206\end{array}$ & $\begin{array}{r}4.1 \\
4.9 \\
5.6 \\
6.5 \\
7.6 \\
8.8 \\
10.0 \\
10.6\end{array}$ & $\begin{array}{l}16 \\
16 \\
16 \\
16 \\
16 \\
16 \\
16 \\
16\end{array}$ & $\begin{array}{l}3.71 \\
3.66 \\
3.62 \\
3.58 \\
3.54 \\
3.51 \\
3.49 \\
3.48\end{array}$ & $\begin{array}{l}4.06 \\
4.31 \\
4.58 \\
4.90 \\
5.26 \\
5.67 \\
6.16 \\
6.43\end{array}$ & $\begin{array}{r}23 \\
43 \\
91 \\
206 \\
542 \\
1688 \\
6510 \\
14129\end{array}$ & $\begin{array}{r}12 \\
20 \\
38 \\
79 \\
187 \\
470 \\
1442 \\
2719\end{array}$ & $\begin{array}{l}c \\
c \\
c \\
c \\
c \\
c \\
c \\
c\end{array}$ & $\begin{array}{l}1 \\
7 \\
1 \\
1 \\
1 \\
1 \\
1 \\
1\end{array}$ \\
\hline \multicolumn{7}{|c|}{ SURFACE FINISH $=72$ RMS } & & & & \\
\hline $\begin{array}{l}95 \\
90 \\
85 \\
80 \\
75 \\
70 \\
65 \\
60 \\
55\end{array}$ & $\begin{array}{l}4.16 \\
4.38 \\
4.64 \\
4.89 \\
5.20 \\
5.54 \\
5.94 \\
6.39 \\
6.92\end{array}$ & $\begin{array}{l}.073 \\
.080 \\
.089 \\
.099 \\
.111 \\
.126 \\
.144 \\
.165 \\
.192\end{array}$ & $\begin{array}{l}7.6 \\
7.5 \\
7.4 \\
7.0 \\
6.5 \\
6.0 \\
5.5 \\
5.0 \\
4.5\end{array}$ & $\begin{array}{l}16 \\
16 \\
16 \\
16 \\
16 \\
16 \\
16 \\
16 \\
16\end{array}$ & $\begin{array}{l}3.54 \\
3.54 \\
3.55 \\
3.56 \\
3.58 \\
3.59 \\
3.62 \\
3.65 \\
3.68\end{array}$ & $\begin{array}{l}3.90 \\
4.10 \\
4.30 \\
4.54 \\
4.81 \\
5.09 \\
5.42 \\
5.79 \\
6.21\end{array}$ & $\begin{array}{r}15 \\
24 \\
44 \\
78 \\
159 \\
348 \\
863 \\
2454 \\
8304\end{array}$ & $\begin{array}{r}8 \\
12 \\
20 \\
35 \\
65 \\
123 \\
260 \\
612 \\
1630\end{array}$ & $\begin{array}{l}c \\
c \\
c \\
c \\
c \\
c \\
c \\
c \\
c\end{array}$ & $\begin{array}{l}1 \\
1 \\
1 \\
1 \\
1 \\
1 \\
1 \\
1 \\
1\end{array}$ \\
\hline
\end{tabular}


MATERIAL

$\underline{A-286}$

FORM

SHEET

CONDITION

ANNEALED \& AGED

SPECIFICATIONS

AMS 5525

DIRECTION

FLEXURE NORMAL TO DIRECTION OF ROLLING

PROPERTY

FLEXURAL FATIGUE STRENGTH @ RT

\begin{tabular}{cccccc}
\hline NO. OF CYCLES & $\begin{array}{c}\text { X } \\
(\text { KSI })\end{array}$ & $k$ & $s$ & $\begin{array}{c}\text { DESIGN } \\
\text { ALLOWABLE } \\
(\text { KSI })\end{array}$ & $\begin{array}{c}\text { DATA } \\
\text { CATEGORY }\end{array}$ \\
\hline
\end{tabular}

A. SURFACE FINISH $=10$ RMS

$\begin{array}{cllll}3.16 \times 10^{6} & 67.5 & 3.50 & 1.57 & 62.0 \\ 10^{6} & 72.5 & 3.52 & 1.68 & 66.6 \\ 3.16 \times 10^{5} & 77.9 & 3.56 & 1.60 & 72.2 \\ 10^{5} & 84.4 & 3.61 & 1.58 & 78.7 \\ 3.16 \times 10^{4} & 92.2 & 3.68 & 1.60 & 86.3\end{array}$

B. SURFACE FINISH $=72$ RMS

$\begin{array}{cllll}3.16 \times 10^{6} & 58.7 & 3.66 & 1.56 & 53.0 \\ 10^{6} & 64.4 & 3.62 & 1.93 & 57.4 \\ 3.16 \times 10^{5} & 70.6 & 3.59 & 1.87 & 63.9 \\ 10^{5} & 78.5 & 3.56 & 1.99 & 71.4 \\ 3.16 \times 10^{5} & 87.8 & 3.55 & 1.92 & 81.0\end{array}$

$\begin{array}{ll}C & 1 \\ C & 1 \\ C & 1 \\ C & 1 \\ C & 1\end{array}$


1.

\section{TEST MATERIAL}

Flexural fatigue testing $(R=-1)$ on $A-286$ annealed and aged sheet was conducted by Rocketdyne as described in Reference (1). Two sheets of the material were polished to two different surface finishes, 10 and $72 \mathrm{rms}$, prior to fabrication of the flexure specimens. The specimens were prepared so that the direction of flexing was normal to the sheet rolling direction. About ten specimens of each surface finish were tested at each of three temperatures, room temperature, $-320^{\circ} \mathrm{F}$ and $-423^{\circ} \mathrm{F}$. Alternating bending stress levels were selected so that failure would generally occur between about $10^{4}$ and $10^{7}$ cycles. The number of replications per stress level ranged from one to three.

\section{DATA ANALYSIS}

The data were analyzed by the method of multiple regression, in which the two independent variables were stress and surface finish, and the dependent variable was the $\log$ of cycle life. Data for the three temperatures were analyzed separately.

The computer program MULEFAT on the GE Time Sharing Computer was used to select regression models, to determine the coefficients of the associated regression equations, the standard error of estimate and the predicted cycle life at each stress leve1. The following models were found to apply at the three temperatures:

$$
\begin{aligned}
& \text { At }-423^{\circ} \mathrm{F} \\
& \qquad Y=A+B X_{1}+C X_{2}+D\left(X_{1} X_{2}\right), \\
& \text { where } Y=10 g \text { of cycle life, } X_{1}=\text { stress level, } X_{2}=\text { surface finish, } \\
& \text { and } A, B, C \text { and } D \text { are the regression coefficients. }
\end{aligned}
$$


DRM: 05.04

DATE: $4-21-71$

PAGE : 9 of 10

$$
\begin{aligned}
& \text { At }-320^{\circ} \mathrm{F} \\
& \quad Y=A+B\left(1 / X_{1}\right)+C X_{2}+D\left(X_{2} / X_{1}\right)
\end{aligned}
$$

At Room Temperature

$$
1 / Y=A+B X_{1}+C X_{2}+D\left(X_{1} X_{2}\right)
$$

The index of determination was $.960, .981$, and .980 at the three temperatures respectively, the high indices denoting excellent fits of the models to the data.

The computer program REGLIM was used to compute the $99 / 95$ limits for $\log$ cycle life and the effective sample size at each stress level. This program is based on a matrix method given in Reference (2). From the effective sample sizes, k-values were obtained by graphical interpolation of the Sandia tables (Reference 3). Because the regression equation for room temperature is in reciprocal form with respect to the dependent variable $(1 / Y)$, the standard error of estimate for $Y$ differs at the various stress levels as seen on page 6 . At the other temperatures, the standard error of estimate is the same at all stress levels. The means and lower limit of cycle life are shown both in logarithmic form and converted back to numbers of cycles.

The means and lower limits were plotted versus stress for each temperature/ surface finish combination. From these plots, mean stress and allowable stress corresponding to various cycle lives were determined (pages $3,5,7$ ). The standard deviations of these estimates were calculated by the differences between the means and lower limits by the k-value corresponding to the mean stress.

The data have been categorized as " $\mathrm{C}$ " because lots are not identified and lot-to-lot variation is not considered. 
DRM: 05.04

DATE: $\quad 4-21-71$

PAGE: 10 of 10

\section{REFERENCES}

(1) Rocketdyne Report R-7564, "Fatigue Properties of Sheet, Bar and Cast Metallic Materials for Cryogenic Applications," dated 30 August 1968.

(2) Draper and Smith, "Applied Regression Analysis," Wiley, 1966, p. 122 .

(3) Owen, D. B., "Factors for One-Sided Tolerance Limits and for Variable Sampling Plans, "Monograph No. SCR-607, Sandia Corporation (1963-1964). 
MATERIAL: A-286

DRM NO. 05.0 .

DATE : $\quad 5-24-71$

PAGE: 1 of 6

AEROJET NUCLEAR SYSTEMS COMPANY

MATERIALS DATA RELEASE

CONTENTS

\begin{tabular}{|c|c|c|c|c|c|c|}
\hline MATERIAL & FORM & CONDITION & DIRECTION & PROPERTY & $\begin{array}{l}\text { DATA } \\
\text { CATEGORY } \\
\end{array}$ & $\begin{array}{l}\text { SOURCE } \\
\text { REFERENCE }\end{array}$ \\
\hline$A-286$ & FORGINGS & $\begin{array}{l}\text { SOL.TREATED } \\
\text { AND AGED }\end{array}$ & TANGENTIAL & ULTIMATE TENSILE STRENGTH & B & 1 \\
\hline$A-286$ & FORGINGS & $\begin{array}{l}\text { SOL. TREATED } \\
\text { AND AGED }\end{array}$ & TANGENTIAL & YIELD TENSILE STRENGTH & B & 1 \\
\hline$A-286$ & FORGINGS & $\begin{array}{l}\text { SOL.TREATED } \\
\text { AND AGED }\end{array}$ & TANGENTIAL & ELONGATION & B & 1 \\
\hline
\end{tabular}

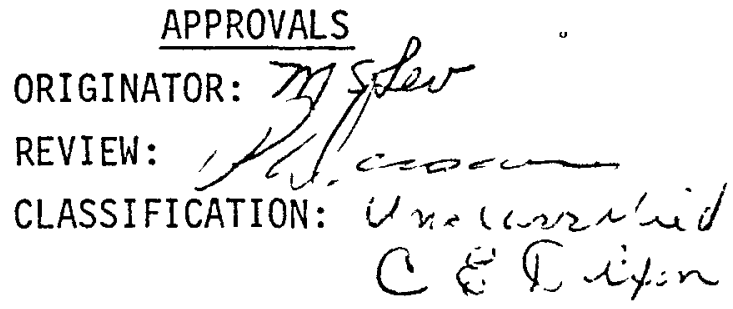


DRY NO: $\quad 65.05$

OA-E: $5-24-71$

PAGE 2 OF 6

DESTG 24-

WEIITLL A-285 FORM FORGINGS CONDITION SOLLTION TREATED AND AGED

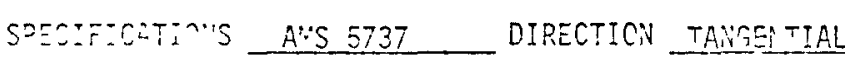

F-YZES-S ח.? OFESE YIELD TENSILE STRENGTH, KSI

\begin{tabular}{|c|c|c|c|c|c|c|c|c|c|c|}
\hline $\begin{array}{ll}\because & c= \\
C & =\end{array}$ & $\begin{array}{l}\text { No. OF } \\
\text { LOTS }\end{array}$ & $\begin{array}{c}\text { "EA: } \\
\text { (ONLFALL) }\end{array}$ & $\begin{array}{l}\text { MEA: OF } \\
\text { LOVEST LOT }\end{array}$ & 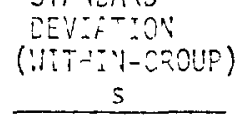 & $M^{M-}$ & $\mathrm{F}^{+}$ & k & $\begin{array}{l}\text { DESTEN } \\
\text { A'LOAABLE }\end{array}$ & $\begin{array}{l}\text { OFTA } \\
\text { CATEGORY }\end{array}$ & $\begin{array}{l}\text { SOLPCE } \\
\text { REFEF-YCE }\end{array}$ \\
\hline$-\because 23$ & 3 & 137.9 & 132.8 & 4.33 & 4 & $2^{-}$ & 3.58 & 122.4 & 8 & 7 \\
\hline$-i^{2}=$ & 3 & 173.0 & $1: 0.5$ & 4.33 & 4 & 21 & 3.58 & 35.3 & $B$ & 1 \\
\hline 6 & 3 & 105.3 & 102.0 & 4.33 & 2 & 23 & 3.83 & 85.2 & $B$ & 1 \\
\hline
\end{tabular}

* $M=S \because$ MLE SIZE FOR MEAN; $F=$ DEGREES OF FREEDOM FOR $s$ 
DRN: $\quad 05.05$

DATE: $5-24$

PAGE 3 OF 6

\section{DESIG.Y DATA}

WATERIAL A-286 FORM FORGINGS

CONDITION

SPECIFICATIONS Al'S 5737 DIRECTION TANGENTIAL

PRODERTY ULTIHATE TENSILE STRENGTH, KSI

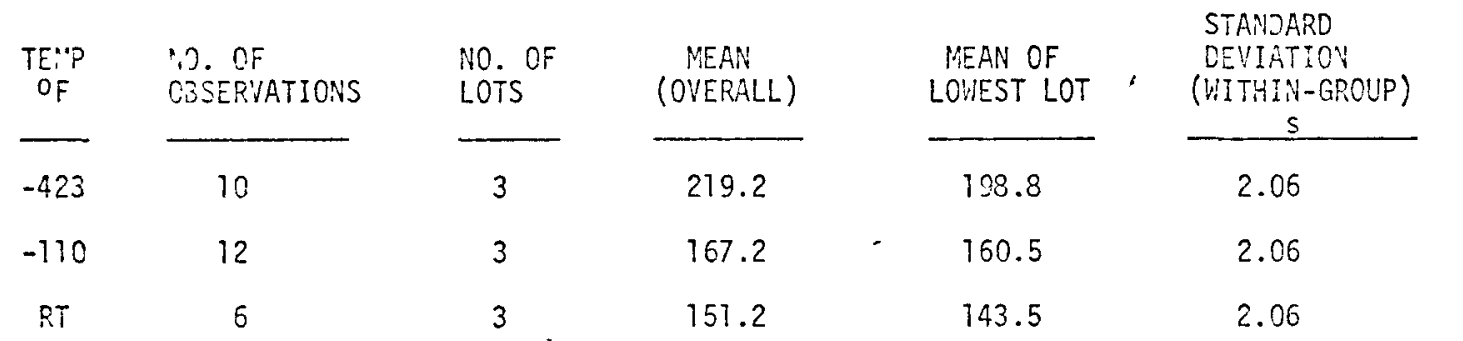

\begin{tabular}{|c|c|c|}
\hline$M^{*}$ & $F^{*}$ & $k$ \\
\hline 2 & 19 & 3.92 \\
\hline 4 & 19 & 3.62 \\
\hline 2 & 19 & 3.92 \\
\hline
\end{tabular}

DESIGN

ALLOWABLE

191.3

153.0

135.4
DATA

CATEGORY

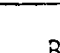

B

B

B
SO'JRCE

REFERENCE

* $. N=$ SAYPLE SIZE FOR MEAN; $F=$ DEGREES OF FREEDOM FOR $s$ 


\section{DESIGN DATA}

IATERIAL FORM

FORGINGS CONDITION SOLUTION TREATED AND AGEO

SFECIFICATIONS A S 5737 DIRECTION
TANGENTIAL

PROFERTY ELONGATION,

\begin{tabular}{|c|c|c|}
\hline $\begin{array}{l}\text { TE" } \\
\text { OF }\end{array}$ & $\begin{array}{l}\text { M. OF. OF } \\
\text { CESERVATIONS }\end{array}$ & $\begin{array}{l}\text { NO. OF } \\
\text { LOTS }\end{array}$ \\
\hline-423 & 10 & 3 \\
\hline-110 & 12 & 3 \\
\hline RT & 6 & 3 \\
\hline
\end{tabular}

\begin{tabular}{|c|c|c|}
\hline $\begin{array}{c}\text { MEAN } \\
\text { (OVERALL) }\end{array}$ & $\begin{array}{l}\text { MEAN OF } \\
\text { LOWEST LOT }\end{array}$ & $\begin{array}{c}\text { STANDARD } \\
\text { DEVIATICN } \\
\text { (WITHIN-GROUP) } \\
\end{array}$ \\
\hline 32.60 & 20.92 & 1.86 \\
\hline 25.58 & 22.75 & 1.85 \\
\hline 20.33 & 17.0 & 1.86 \\
\hline
\end{tabular}

\begin{tabular}{|c|c|c|}
\hline$M *$ & $F^{*}$ & $k$ \\
\hline 2 & 19 & 3.92 \\
\hline 4 & 19 & 3.62 \\
\hline 2 & 19 & 3.92 \\
\hline
\end{tabular}

\begin{tabular}{|c|}
\hline $\begin{array}{l}\text { DESIGIN } \\
\text { ALLOVIABLE }\end{array}$ \\
\hline 13.6 \\
\hline 16.0 \\
\hline 9.7 \\
\hline
\end{tabular}

DATA

CATEGORY

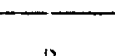

B

13

B
SOURCE REFERENCE

* $i 4=$ SAMPLE SIZE FOR MEAN; $F=$ QEGREES OF FREEDOM FOR $s$ 
DRM 05.05

DATE : $5-24-71$

Page 5 of 6

\section{TEST MATERIAL}

Three forgings, $13^{\prime \prime}$ diameter $\times 3$ 1/2" thick, were made by Coulter Steel and Forge Company from three heats of A-286 (Nos. 31666, 32073 and 76053). Two tensile specimens from each heat were tested at room temperature by the vendor, and four from each heat were tested at -110 and $-423^{\circ} \mathrm{F}$ at the ALRC Materials Laboratory. All tensile specimens were tangentially oriented. 2. DATA ANALYSIS

The tensile data were analyzed according to the methods of Reference (2). The Bartlett-Box test indicated that within-group variances of ultimate strength and elongation at $-423^{\circ} \mathrm{F}$ were not homogeneous. Two of the specimens from heat 31666 were extremely low in these properties in comparison with the other two specimens, although their yield strength was normal. There was no evidence of high variability of this heat at the other temperatures. It was concluded that the low values were probably due to specimen anomalies rather than to non-uniformity of the heat. After the low values were excluded, the within-group variances were homogeneous (for all properties) over al1 three heats and all three temperatures. Accordingly these variances were pooled.

Because of the relatively high heat-to-heat variance, the design allowables calculated by the standard method of Reference (2) were too low to be usable. Instead, the alternate method described in Para. 5.8.3.3 of Reference (2) was used. In this method the lowest lot mean was decreased by an amount $k s$, where $s$ is the pooled within-lot standard deviation and $k$ is the appropriate 99/95 tolerance limit factor. For the purpose of estimating the lowest lot mean, the discrepant values were returned to the data. 
DRM $\quad 05.05$

DATE : $5-24-71$

Page 6 of 6

The design allowables are classified as " $B$ " data because of the use of an alternate method. (Ref. (3)).

\section{REFERENCES}

*(1) Memorandum N8130-0031 from P. P. Dessau to M. S. Lev dated May 1971, Subject: A-286 Tensile Data

(2) NERVA Program Procedure, R101-NRP-503, "Statistical Analysis of Materials Test Data"

(3) Materials Memorandum 70-320, dated 25 August 1970, from C. W. Funk to I. L. Odgers, Subject: "Material Properties Data Book Meeting - SNPO-C, 18-19 August 1970." (Includes "Statement of Understanding of Treatment of Anomalies in P1anned "A" Data", signed by representatives of ANSC, WANL, and SNPO-C)

* Data to be included in forthcoming Materials Quarterly Report for 3rd quarter, CY 71. 
AEROJET NUCLEAR SYSTEMS COMPANY

MATERIALS DATA RELEASE

CONTENTS

$\begin{array}{ll}\text { MATERIAL } & \text { CONDITION } \\ \text { AGCarb 101 } & \text { UNIRRADIATED \& IRRADIATED } \\ \text { AGCarb } 101 & \text { UNIRRADIATED \& IRRADIATED } \\ \text { AGCarb } 101 & \text { UNIRRADIATED \& IRRADIATED } \\ \text { AGCarb } 101 & \text { UNIRRADIATED \& IRRADIATED } \\ \text { AGCarb } 101 & \text { UNIRRADIATED \& IRRADIATED }\end{array}$

SYMBOLS USED ON PAGES 2-6

$\bar{X}=$ GROUP AVERAGE

$n=$ SAMPLE SIZE ASSOCIATED WITH $\bar{x}$

$f=$ DEGRESS OF FREEDOM FOR POOLED WITHIN-GROUP STANDARD DEVIATIONS

$k=99 / 95$ ONE-SIDED TOLERANCE LIMIT FACTOR FOR $n$ AND $f$.

$s=$ POOLED WITHIN-GROUP STANDARD DEVIATION.

$D A=$ DESIGN ALLOWABLE $=\bar{x}-k S$ (ALSO FOR MODULUS, $\bar{x}+k s$ )

\section{APPROVALS}

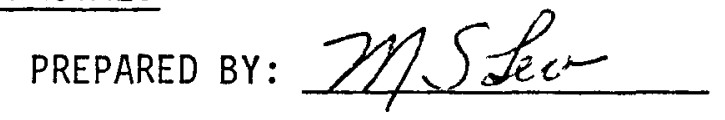

REVIEW:

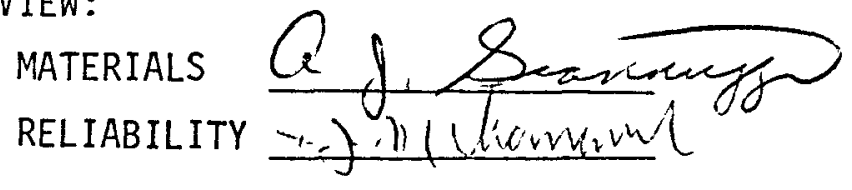

\begin{tabular}{lcc} 
PROPERTY & DATA CATEGORY & PAGE \\
\cline { 2 - 3 } TENSILE STRENGTH & $C$ & 2 \\
FLEXURAL STRENGTH & $\mathrm{C}$ & 3 \\
FLEXURAL MODULUS & $\mathrm{C}$ & 4 \\
HARDNESS (ROCKWELL R) & $\mathrm{C}$ & 5 \\
YOUNG'S MODULUS & $\mathrm{C}$ & 6
\end{tabular}


DESIGN DATA

Ob. 44

DATE : 2 JULY 1971

PAGE: 2 OF 12

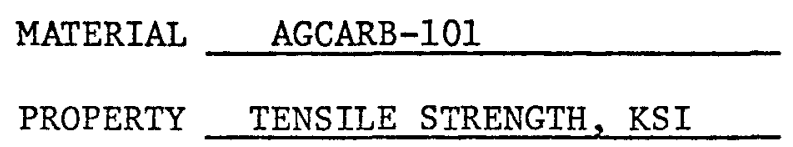

TEMPERATURE

RT

\begin{tabular}{|c|c|c|c|c|c|c|c|c|}
\hline CONDITION & $\overline{\mathrm{X}}$ & $n$ & $i$ & $k$ & $s$ & DA & $\begin{array}{l}\text { DATA } \\
\text { CATEGORY }\end{array}$ & $\begin{array}{l}\text { SOURCE } \\
\text { REFERENCE }\end{array}$ \\
\hline UNIRRADIATED (1) & 9.24 & 6 & 15 & 3.63 & 1.28 & 4.6 & $\mathrm{C}$ & (1) \\
\hline IRRADIATED ${ }^{(2)}$ & 11.18 & 6 & 15 & 3.63 & 1.28 & 6.5 & $\mathrm{C}$ & (1) \\
\hline IRRADIATED + ANNEAI (3) & 10.16 & 12 & 15 & 3.50 & 1.28 & 5.7 & $\mathrm{C}$ & (1) \\
\hline
\end{tabular}

NOTES :

(1) INCLUDES UNANNEALED SPECIMENS AND THOSE ANNEALED 1 HOUR AT 1760 AND 3000 R; (EFFECT OF ANNEALING NOT SIGNIFICANT)

(2) $5 \times 10^{17} \mathrm{AND} 5 \times 10^{18} \mathrm{NVT}(\mathrm{E}>1 \mathrm{MeV})$

(3) LISTED AS SEPARATE ITEM BECAUSE RECOVERY UPON ANNEALING WAS NOT QUITE COMPLETE. 
DRM 06.04

DATE: 2 JULY 1971.

PAGE: 3 OF 12

MATERIAL

PROPERTY

AGCARB-101

FLEXURAL STRENGTH, KSI

TEMPERATURE

RT

\section{CONDITION}

UNIRRADIATED (1)

IRRADIATED (2)

$\begin{array}{ccccccc}\frac{\bar{x}}{13.28} & \frac{n}{18} & \frac{f}{15} & \frac{k}{3.45} & & \frac{s}{0.92} & \frac{\mathrm{DA}}{10.1} \\ 16.40 & 6 & 15 & 3.63 & 0.92 & 13.1\end{array}$

DATA

CATEGORY

C

C
SOURCE REFERENCE

NOTES :

(1) INCLUDED UNANNEALED SPECIMENS AND THOSE ANNEALED $1 \mathrm{HR}$. AT $1760^{\circ}$ AND $3000^{\circ} \mathrm{R}$ (NO SIGNIFICANT CHANGE ON ANNEALING); ALSO INCLUDES IRRADIATED + ANNEALED GROUP (PROPERTY COMPLETELY RECOVERED ON ANNEALING).

(2) $5 \times 10^{17}$ AND $5 \times 10^{18} \mathrm{NVT}(\mathrm{E}>1 \mathrm{MeV})$ 
DESIGN DATA

DRM 06.04

DATE: 2 JULY 1971.

PAGE: 4 OF 12

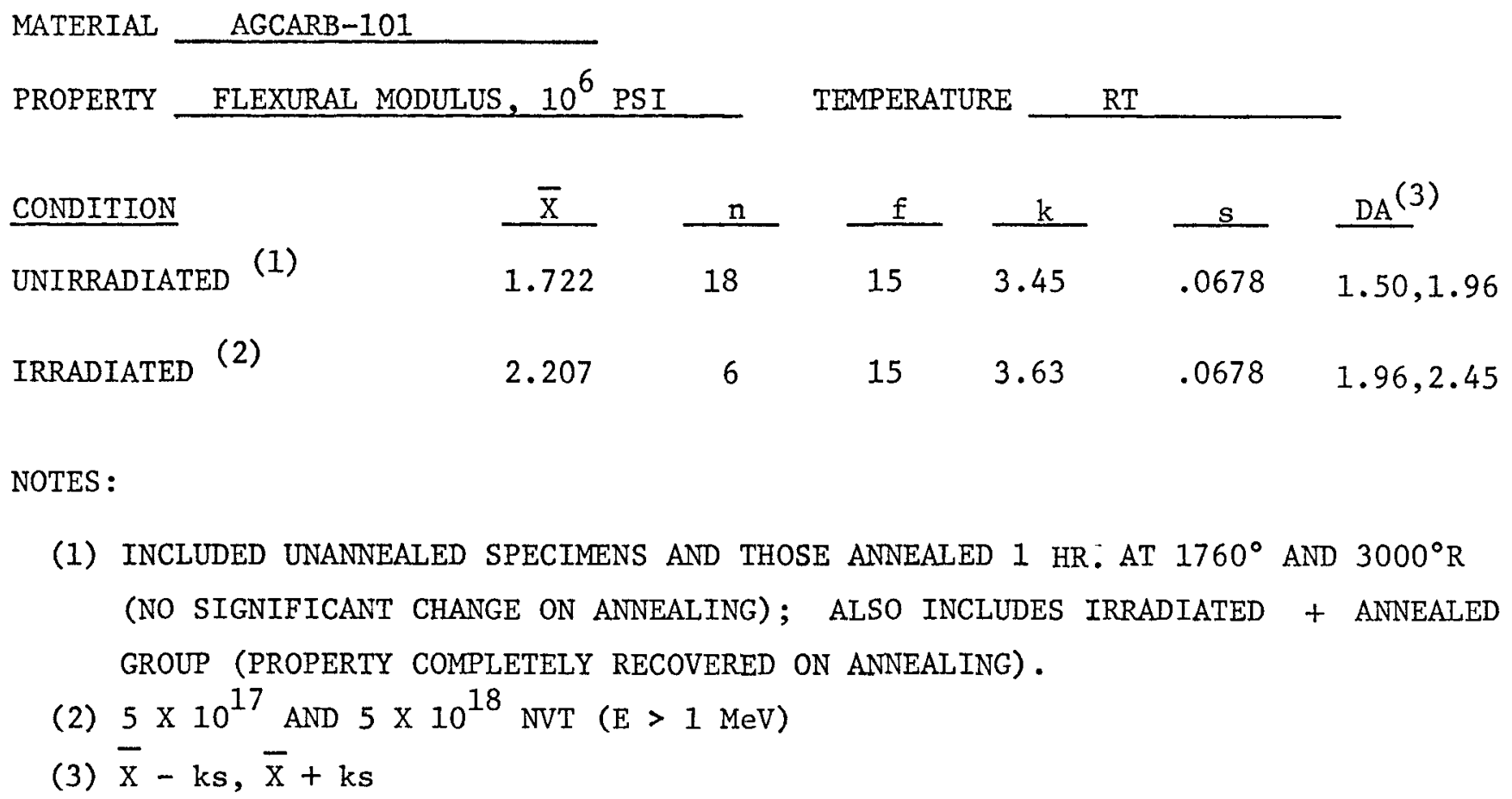

TEMPERATURE RT

CONDITION

UNIRRADIATED (1)

IRRADIATED（2）

$\begin{array}{cc}\frac{\overline{\mathrm{X}}}{1.722} & \frac{\mathrm{n}}{18} \\ 2.207 & 6\end{array}$

NOTES :

(1) INCLUDED UNANNEALED SPECIMENS AND THOSE ANNEALED 1 HR: AT $1760^{\circ}$ AND $3000^{\circ} \mathrm{R}$ (NO SIGNIFICANT CHANGE ON ANNEALING); ALSO INCLUDES IRRADIATED + ANNEALED GROUP (PROPERTY COMPLETELY RECOVERED ON ANNEALING) .

(2) $5 \times 10^{17} \mathrm{AND} 5 \times 10^{18} \mathrm{NVT}(\mathrm{E}>1 \mathrm{MeV})$

(3) $\bar{X}-k s, \bar{X}+k s$

\begin{tabular}{|c|c|c|c|c|c|}
\hline$f$ & $\mathrm{k}$ & $s$ & $\mathrm{DA}^{(3)}$ & $\begin{array}{l}\text { DATA } \\
\text { CATEGORY }\end{array}$ & $\begin{array}{l}\text { SOURCE } \\
\text { REFERENCE }\end{array}$ \\
\hline 15 & 3.45 & .0678 & $1.50,1.96$ & C & (1) \\
\hline 15 & 3.63 & .0678 & $1.96,2.45$ & C & (1) \\
\hline
\end{tabular}


DRM 06.04

DATE: 2 JULY 1971

PAGE: 5 OF 12

DESIGN DATA

MATERIAL

AGCARB-101

PROPERTY

HARDNESS (ROCKWELL R)

TEMPERATURE

$\mathrm{RT}$

CONDITION

UNANNEALED ${ }^{(1)}$

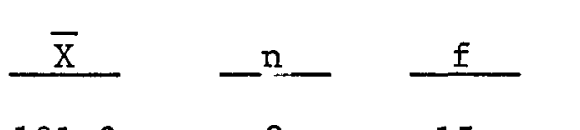

101.2

8

15

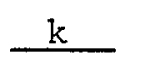

3.57

S DA

DATA

CATEGORY

ANNEALED (1), (2)

$96.5 \quad 16$

$15 \quad 3.47$

3.41

89

C

SOURCE

REFERENCE

(1)

(1)

NOTES :

(1) INCLUDES BOTH IRRADIATED AND UNIRRADIATED GROUPS (NO SIGNIFICANT EFFECT OF IRRADIATION)

(2) INCLUDES $1760^{\circ}$ AND $3000^{\circ} \mathrm{R} I \mathrm{HR}$ TREATMENTS 
DESIGN DATA

MATERIAL

PROPERTY YOUNG'S MODULUS, $10^{6}$ PSI TEMPERATURE RT

\begin{tabular}{|c|c|c|c|c|c|c|c|c|}
\hline CONDITION & $\overline{\mathrm{X}}$ & $\mathrm{n}$ & $\mathrm{f}$ & $k$ & $s$ & $\mathrm{DA}^{* *}$ & $\begin{array}{l}\text { DATA } \\
\text { CATEGORY } \\
\end{array}$ & $\begin{array}{l}\text { SOURCE } \\
\text { REFERENCE }\end{array}$ \\
\hline UNIRRADIATED & 2.92 & 4 & 3 & 7.0 & .044 & $2.6,3=2$ & C & (4) \\
\hline IRRADIATED* & 3.65 & 2 & 3 & 7.4 & .044 & $3.3,4.0$ & $\mathrm{C}$ & (4) \\
\hline
\end{tabular}

* 1 SPECIMEN AT $5 \times 10^{17}$ AND 1 SPECIMEN AT $5 \times 10^{18}$ NVT

$* * \bar{x}-\mathrm{ks}, \overline{\mathrm{X}}+\mathrm{ks}$ 
DRM 06.04

DATE: 2 JULY 197]

1. TEST DESCRIPTION

AGCARB-101 tensile and flexural specimens were irradiated in test GTR-20C in order to determine the effects of radiation upon their mechanical properties and the possible reversal of these effects upon annealing. (References 1, 2, 3) The test matrix, a $3 \times 3$ factorial design, was as follows:

FLUENCE, NVT $(E>1 \mathrm{MeV})$

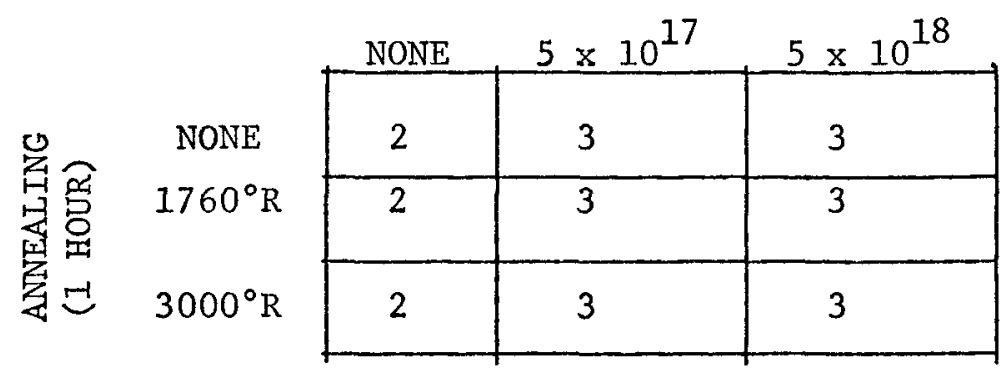

Identical matrices were used for the tensile and flexural specimens. Data were recorded for the following properties:

1. Tensile strength, Ksi

2. Flexural strength, Ksi

3. Flexural modulus, $10^{6}$ psi

4. Hardness (Rockwell R)

Specimens were randomly assigned to the various experimental conditions and tested in random order. Testing procedure followed Federal Test Method No. 406. All specimens were tested at room temperature. 
DRM 06.04

DATE: 2 JULY 1971

PAGE: 8 OF 12

\section{DATA ANAIYSIS}

For all four properties the within-cell variances were found to be homogeneous according to the Bartlett-Box test at the .10 significance level. Pooling of these variances was therefore justified. Averages for each cell of the matrix are shown in the following table.

\begin{tabular}{l|l|c|c|c|}
\multirow{2}{*}{} & & UNIRRADIATED & \multicolumn{2}{|c|}{ IRRADIATED } \\
\cline { 2 - 5 } & Anneal & & $5 \times 10^{17}$ & $5 \times 10^{18}$ \\
\cline { 2 - 5 } Tensile & None & 8.86 & 11.61 & 10.75 \\
Strength & $1760^{\circ} \mathrm{R}$ & 8.86 & 10.54 & 9.97 \\
& $3000^{\circ} \mathrm{R}$ & 10.01 & 9.38 & 10.75 \\
\cline { 2 - 5 } Flexura1 & None & 14.07 & 17.20 & 15.59 \\
Strength & $1760^{\circ} \mathrm{R}$ & 13.88 & 13.34 & 13.68 \\
& $3000^{\circ} \mathrm{R}$ & 12.06 & 12.58 & 13.45 \\
\cline { 2 - 5 } Flexura1 & None & 1.74 & 2.22 & 2.19 \\
Modulus & $1760^{\circ} \mathrm{R}$ & 1.70 & 1.69 & 1.84 \\
& $3000^{\circ} \mathrm{R}$ & 1.72 & 1.71 & 1.72 \\
\hline Hardness & None & 99.5 & 99.5 & 104.0 \\
Rockwe11 "R" & $1760^{\circ} \mathrm{R}$ & 95.8 & 98.2 & 97.0 \\
\cline { 2 - 5 } & $3000^{\circ} \mathrm{R}$ & 93.0 & 96.7 & 97.0 \\
\cline { 2 - 5 } & & & &
\end{tabular}

Analysis of variance, applied to the irradiated data only, showed that there were no significant differences between the two fluence levels. Similarly, when applied to the annealed data only showed that there were no significant differences between the effects of the two annealing temperatures. Consequently, the original matrix was reduced, for the purpose of further analysis, to the following $2 \times 2$ array:

\begin{tabular}{|l|c|c|}
\hline \multicolumn{1}{l|}{} & UNIRRADIATED & IRRADIATED \\
\hline Unannealed & $2(\mathrm{~A})$ & $6(\mathrm{~B})$ \\
\hline Annealed & $4(\mathrm{C})$ & $12(\mathrm{D})$ \\
\hline
\end{tabular}


DRM 06.04

DATE: 2 JULY 1971

PAGE: 9 OF 12

Average properties for each of the four cells of this matrix are plotted in Figure 1. This diagram shows that there was an increase for each property upon irradiation and that annealing tended to reverse this effect. To assess the statistical significance of these changes, four distinct comparisons were defined and tested by means of t-tests on the cell averages with the following results:

(The t-tests were based on the pooled within-group variances with 15 , degrees of freedom.)

COMPARISON

Irradiated vs Unirradiated Annealing of Unirradiated Annealing of Irradiated Net Effect (Irrad.+ Anneal)

\section{DEF INITION}

$\mathrm{B}-\mathrm{A}$

$\mathrm{C}-\mathrm{A}$

$\mathrm{B}-\mathrm{D}$

D-A
SIGNIFICANT CHANGE AT .05 LEVEL? Tensile Flexural Flexural HardStrength Strength Modulus ness

Yes Yes Yes No

No No No Yes

No Yes Yes Yes

No * No * No * No *

* "No" in this case means recovery of original properties.

The first three properties exhibited essentially the same pattern, illusstrated best by the Flexural Modulus, (See Figure 1) for which the change upon radiation is completely reversed by annealing. Hardness differed in that annealing alone caused a statistically significant reduction in both the irradiated and unirradiated condition; the effect of irradiation alone was not significant. 
DRM 06.04

DATE: 2 JULY 1971

PAGE: 10 OF 12

The groupings shown in the tables of Pages 2, 3, 4 and 5 are based upon the results of these significance tests. The pooled standard deviations were used along with the appropriate cell averages (pooled if warranted) to calculate the $99 / 95$ design allowables, $\overline{\mathrm{X}}$ - ks.*

A few Young's modulus determinations were also made on both irradiated and unirradiated specimens. (Reference 4) Although the data were insufficient for complete statistical treatment, the average and design allowables* (Page 6) are also classified as " $\mathrm{C}$ ".

* For modulus (flexural and Young's) both the upper and lower 99/95 1imits are given as design allowables, to be used at the discretion of the designer. 


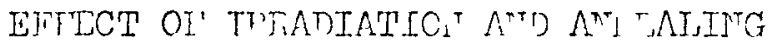

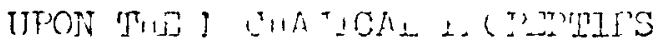

OF $\triangle \mathrm{GCarb}-101$
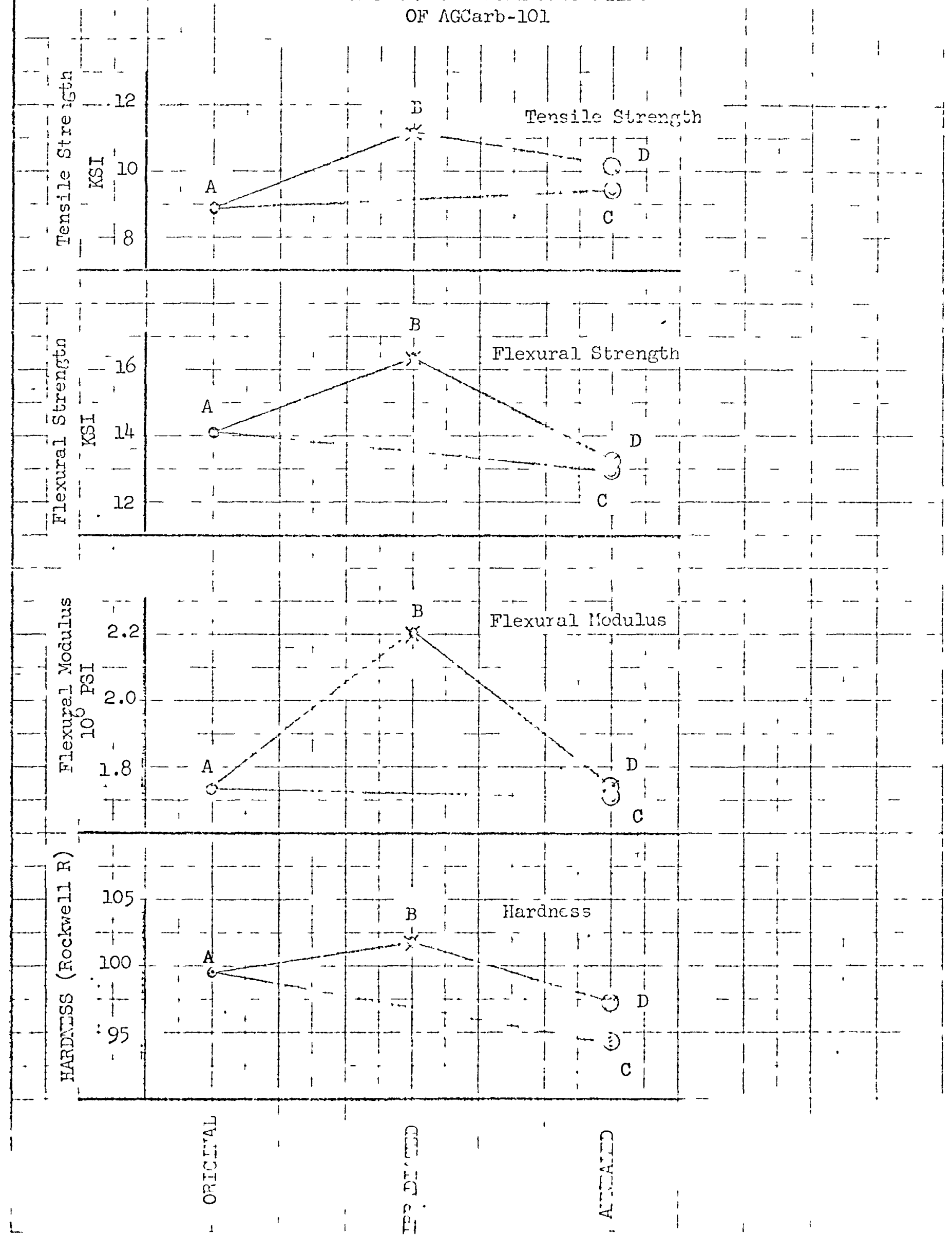
DRM 06.04

DATE: 2 JULY 1971

PAGE: 12 OF 12

REFERENCES :

1. Materials Memorandum 71-115, A. J. Giannuzzi to C. E. Dixon, Subject: The Effect of Irradiation on the Tensile Strength, Flexural Strength, Flexural Modulus and Hardness of AGCarb-101, dated 25 March 1971.

2. Materials Memorandum 71-006, A. J. Giannuzzi to C. E. Dixon, Subject: Test Program for AGCarb-101 Tensile and Flexural Specimens Irradiated in Test GTR-20C, dated 20 January 1971.

3. GTR 20C Data Report for AGCarb-101, General Dynamics, Convair Aerospace Division, dated 10 November 1970.

4. Materials Memorandum 71-088, A. J. Giannuzzi to C. E. Dixon, Subject: The effect of Irradiation on Young's Modulus and on the Dimensions of AGCarb-101, dated 26 February 1971. 